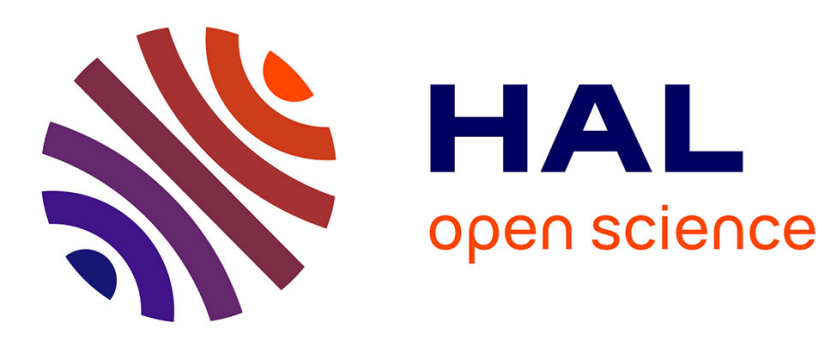

\title{
Evidential networks for reliability analysis and performance evaluation of systems with imprecise knowledge
}

Christophe Simon, Philippe Weber

\section{To cite this version:}

Christophe Simon, Philippe Weber. Evidential networks for reliability analysis and performance evaluation of systems with imprecise knowledge. IEEE Transactions on Reliability, 2009, 58 (1), pp.69-87. 10.1109/TR.2008.2011868 . hal-00340040

\author{
HAL Id: hal-00340040 \\ https://hal.science/hal-00340040
}

Submitted on 19 Nov 2008

HAL is a multi-disciplinary open access archive for the deposit and dissemination of scientific research documents, whether they are published or not. The documents may come from teaching and research institutions in France or abroad, or from public or private research centers.
L'archive ouverte pluridisciplinaire HAL, est destinée au dépôt et à la diffusion de documents scientifiques de niveau recherche, publiés ou non, émanant des établissements d'enseignement et de recherche français ou étrangers, des laboratoires publics ou privés. 


\title{
Evidential networks for reliability analysis and performance evaluation of systems with imprecise knowledge
}

\author{
Christophe SIMON and Philippe WEBER
}

\begin{abstract}
This paper deals with evidential networks to manage imprecise probabilities. We also extend utility functions to evidential networks. We apply evidential networks in reliability analysis with imprecise parameters and evaluate system performance with imprecise probabilities and utility functions. Two examples are analyzed to show the interest of the proposed method.
\end{abstract}

Index Terms-Imprecise probability, Dempster-Shafer Theory, Utility functions, credal networks.

\section{INTRODUCTION}

$\mathbf{T}$ HE reliability study of systems is a major part of process control in companies. Many methods exist for quantitative analyses of the reliability of systems and many of them are perfectly referred. Such methods like Fault trees, Markov chains and simplified equations are quoted in standards [1]-[3] or are the subject of a standard [4].

The study of system reliability is now an old science, so, the developments and the improvements of existing analysis tools offer broad possibilities of modeling compared to the tools referred in these standards [1]-[3]. For instance, we can quote the dynamic fault trees, stochastic Petri nets, homogeneous or non-homogeneous Markovian processes, binary decision diagrams, Monte Carlo simulations [5], [6], ...

In our opinion, Bayesian networks are an innovating tool for the study of system dependability. They allow graphical approach to the functioning and malfunctioning of systems and allow computing of reliability and other parameters. They offer a compact, modular approach and can be instantiate [7] that gives them many powerful modeling capacities. Moreover, many authors showed the equivalence with the standard tools under certain assumptions [8]. These various characteristics made Bayesian networks a tool of interest and their improvements and applications are important in many fields [9].

In reliability studies of systems, the probabilities are usually considered as precise and perfectly determinable. Moreover, all the information on the behavior of reliability of a system and its components is available. This completeness assumes two essential conditions [10]:

- All the probabilities or probability distributions are known perfectly.

- The system components are independent, i.e. each random variables describing the components' reliability are independent or their dependences are known precisely.

C. Simon and Ph. Weber are with the Research Center on Automatic Control of Nancy,Nancy-university, CNRS, France.
Utkin [10] wrote that the first condition is rarely fulfilled and advocates the use of probability intervals to manage this problem [11]. We agree with this analysis but consider that the use of imprecise probabilities as intervals is just one of the multiple ways to handle the problem of precision in the knowledge of probabilities. Indeed, many other authors considered the problem of precision with probabilities densities [12], envelop of probabilities [13], imprecise probabilities [14], fuzzy numbers [15]-[18] or belief functions [19]. In our opinion, the question is not to oppose these various methods but rather to choose correctly the framework of study in which they have the best assets [20].

When the components reliability is computed from databases, the problem of imprecision is critical and can be easily handled by a probability distribution if the amount of data is sufficient. However, the problem of incoherency and completeness of data cannot be suitably handled by the probability theory. Indeed, these problems cannot be viewed as an uncertainty on the value of a probability but like a problem of uncertainty on the state of the components, an epistemic uncertainty. Thus, the theory of evidence proposes an interesting and suitable formalism to handle this type of uncertainty.

The theory of evidence also called the theory of the belief functions is rather close to the theory of probability on certain points. On one hand, this closeness is interesting for engineers in reliability. On the other hand, the theory suffers from a major drawback in its use in complicated contexts. Indeed, the assignment function of the elementary belief masses, which can be compared to the elementary probabilities on the subsets of a random variable, requires allocating all the belief masses to each combination of the states of affairs, 1 to 1,2 to 2 ... Since the number of state of affairs is important, the operation becomes tedious. However, this operation can be singularly reduced in the case of the reliability analysis. Thus, we show in this paper how it is possible to simply apply the evidence theory to the reliability analysis and the analysis of performance of multi-states systems.

The goal of this article is to propose an evidential network for the study of reliability and of the performance of systems by the use of junction tree inference algorithms as it was proposed by Simon and Weber [21], [22]. Much work exists around the concept of credal networks applied to the analysis of the reliability or other problems of knowledge management by taking account of epistemic uncertainty. Shenoy et. al developed 'Valuable Networks' [23], [24]. They are pow- 
erful tools and well suited to the modeling of knowledge. In addition, equivalences with the Bayesian networks under certain conditions were proven [25]. Smets [26] also proposed a similar graphic formalism for the handling of uncertain knowledge in the epistemic meaning. Cozman [27], [28] proposes a kind of credal network for the modeling of the imprecise probabilities by networks. Recently, Ben Yaghlane proposes evidential networks based on the transferable belief model of Smets with Dempster-Shafer rule of combination and binary joint tree [29].

To show the handling of epistemic uncertainty by a network approach, we structured our article as follows. In the second section, we give the useful bases of the evidence theory. The third section is dedicated to the formalization of the evidential networks for the handling of imprecise probabilities. We specify the use of the utility functions for the evaluation of performances of multi-states systems. The fourth section is dedicated to the evaluation of the probist reliability and the performance of the systems. The last section is devoted to two case studies in order to show the interest of our approach.

\section{USEFUL BASICS OF EVIDENCE THEORY}

The evidence theory was initiated by Dempster [30] with its work on the upper and lower bounds of a family of probability distributions then reinforced by Shafer [31]. Several models of imperfect data processing were proposed:

- Upper and lower probabilities [32];

- Theory of Dempster-Shafer [31];

- The Hint Model of Kholas and Monney [33];

- The Transferable belief model of Smets [34].

On a discrete finite space, the model suggested by Dempster Shafer can be interpreted as a generalization of the theory of probability where the probabilities are assigned to sets in opposition to singletons mutually exclusive [35]. In the theory of probability, a measure is assigned to only one possible event. On the other hand, in the theory of Dempster-Shafer, a measure can be assigned to a set of events. However, when the information available allows the assignment of measures to single events (i.e. specific knowledge), the model of DempsterShafer merges with the traditional formulation of the probabilities and there are known as Bayesian evidence [36]. The closeness between these two models reinforces the interest of the Dempster-Shafer theory for applications initially handled by the theory of probability.

\section{A. Basic mass assignment}

The main idea of the basic mass assignment is to allocate a measure between 0 and 1 to indicate the degree of belief about an event or assumption [31]. There can be several interpretations of these measurements which generate controversy on their use. The Dempster-Shafer theory doesn't make the assumption of an unknown probability measurement but subjective beliefs based on nonspecific information [37]. In the same way, Sentz [35] argues that it is not really a question about probabilities. However, many works are directed towards an objectivist approach of belief functions [38], [39]. Thus, the term of basic probability assignment as well as that of basic mass assignment are both commonly read in literature [40] to model the same assignment process. The theory of probability as well as the theory of Dempster-Shafer offers an objective point of view or a subjective point of view of knowledge [37]. When the process is carried out on large volumes of data or directly starting from probabilities, the term of basic probability assignment could be perhaps preferred [38], [39]. Basic mass assignment is suitable in the treatment of knowledge from experts' opinions [41]. Duong [37, p.70] argues about the unimportance of this interpretation problem, which occults a mathematical unit. For our part, we will use the term of belief masses in this article.

In the Dempster-Shafer theory, we consider a set of $q$ elements mutually exclusive and exhaustive called the frame of discernment defined by:

$$
\Omega=\left\{H_{1}, H_{2}, \ldots, H_{q}\right\}
$$

$\Omega$ is the finite set of all possible issues where each proposition or hypothesis $H_{i}$ can support any information from different sources. The sources of information can distribute masses on every subset of the frame of discernment:

$$
\begin{array}{r}
A_{i} \in 2^{\Omega}:\left\{\emptyset, A_{1}=\left\{H_{1}\right\}, \ldots, A_{q}=\left\{H_{q}\right\}, A_{q+1}=\left\{H_{1}, H_{2}\right\},\right. \\
\left.\ldots, A_{2^{q}-1}=\left\{H 1, \ldots, H_{q}\right\}\right\}
\end{array}
$$

A source of information assigns a belief mass between 0 and 1 only on hypotheses $A_{i}$ on which it has a direct knowledge, i.e. it does not assign any belief mass to any subset of $A_{i}$ [42] :

$$
0 \leq m\left(A_{i}\right) \leq 1
$$

This process called basic mass assignment is represented by a function $m$ defined by:

$$
m: 2^{\Omega} \rightarrow[0,1]
$$

such as:

$$
m(\emptyset)=0
$$

and

$$
\sum_{A_{i} \in 2^{\Omega}} m\left(A_{i}\right)=1
$$

Each $A_{i}$ supporting $m\left(A_{i}\right)>0$ is called a focal set. The constraint defines on $\emptyset$ by 5 is not mandatory. It supposes that all hypotheses $H_{i}$ are known i.e. we are in the context of closed world assumption. The goal of $\emptyset$ is to formalize that all hypotheses are not known. In this case, $m(\emptyset) \neq 0$ supports this consideration [43].

\section{B. Belief and plausibility measures}

From a belief mass distribution, the upper and lower bounds of a probability interval can be defined. This interval contains the probability of a set of hypotheses or focal sets and is bounded by two non-additives measures called belief $(\mathrm{Bel})$ and plausibility $(P l s)$ [44].

The measure of belief $\operatorname{Bel}\left(A_{i}\right)$ is the lower bound of a focal set $A_{i}$. It is defined as the sum of the belief masses of all subsets $B$ that contribute to $A_{i}$ such as $B \subseteq A_{i}$. The upper bound $P l s\left(A_{i}\right)$ is the sum of all belief masses assigned to 


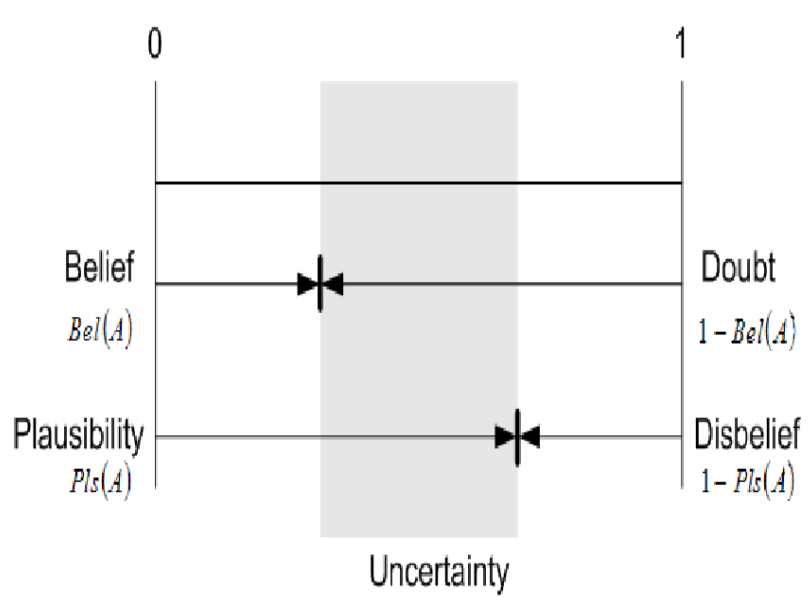

Fig. 1. Plausibility and belief measures and their complement [48]

subsets $B$ such as $B \cap A_{i} \neq \emptyset$. Pls $\left(A_{i}\right)$ and $\operatorname{Bel}\left(A_{i}\right)$ are defined by the following equations:

$$
\begin{gathered}
P l s\left(A_{i}\right)=\sum_{B \mid A_{i} \cap B \neq \emptyset} m(B) \\
\operatorname{Bel}\left(A_{i}\right)=\sum_{B \mid B \subseteq A_{i}} m(B)
\end{gathered}
$$

It results in the bounding property defined by the following equation:

$$
\operatorname{Bel}\left(A_{i}\right) \leq \operatorname{Pr}\left(A_{i}\right) \leq \operatorname{Pls}\left(A_{i}\right)
$$

where $\operatorname{Pr}\left(A_{i}\right)$ defines the occurence probability of $A_{i}$ but remains unknown. It can take any value in $\left[\operatorname{Bel}\left(A_{i}\right), P l s\left(A_{i}\right)\right]$. The bounding property 9 is well known and has been defined since 1976 in the work of Shafer [31]. Many authors used it to connect the interval defined by $\left[\operatorname{Bel}\left(A_{i}\right), P l s\left(A_{i}\right)\right]$ and the belief mass distribution [45]-[47].

Plausibility and belief measures are not dual because they are not additive within the meaning of the probability theory $\left(\operatorname{Bel}(A) \neq P l s\left(A^{c}\right)\right)$ where $A^{c}$ is the complement of $A$ according to $\Omega$. However, the relations below can be established between $A$ and $A^{c}$ :

$$
\operatorname{Bel}\left(A_{i}^{c}\right)=1-\operatorname{Pls}\left(A_{i}\right)
$$

and

$$
\operatorname{Pls}\left(A_{i}^{c}\right)=1-\operatorname{Bel}\left(A_{i}\right)
$$

with

$$
\operatorname{Bel}\left(A_{i}^{c}\right) \leq \operatorname{Pls}\left(A_{i}^{c}\right)
$$

$\left(P l s\left(A_{i}\right)-\operatorname{Bel}\left(A_{i}\right)\right)$ describes the uncertainty concerning hypothesis $A_{i}$ represented by interval $\left[\operatorname{Bel}\left(A_{i}\right), P l s\left(A_{i}\right)\right]$ (cf. figure 1).

From plausibility and belief measures, we obtain the basic mass assignment by the möbius transform [49]:

$$
m\left(A_{i}\right)=\sum_{B \mid B \subseteq A_{i}}(-1)^{\left|A_{i}\right|-|B|} \operatorname{Bel}(B)
$$

where $\left|A_{i}\right|$ is the cardinal of set $A_{i}$.

\section{P-box and belief mass assignment}

Ferson et al. [50] argues that each frame of discernment or each Dempster-Shafer structure specifies a unique p-box (probability box) and that each p-box specifies an equivalent class of Dempster-Shafer structure [51], [52]. Ferson [53] describes the relation between these two generalizations of probability distributions. P-boxes are sometimes considered as a granular approach of imprecise probabilities [32] which are arbitrarily sets of probability distributions.

If the imprecision of a probability measure is described by a p-box, the relation with a basic mass assignment is directly obtained by:

$$
\left[\underline{P}_{H_{i}}, \bar{P}_{H_{i}}\right]=\left[\operatorname{Bel}\left(H_{i}\right), \operatorname{Pls}\left(H_{i}\right)\right]
$$

where $\underline{P}_{H_{i}}$ is the lower probability of hypothesis $H_{i}, \bar{P}_{H_{i}}$ is the upper probability with $i$ from 1 to $q$ the number of hypotheses. The transformation of a set of probability intervals $[P]$ to a basic belief assignment $M$ is obtained easily by the equations 7, 8 and 13. If :

$$
\left[P_{X}\right]=\left[\left[\underline{P}_{H_{1}}, \bar{P}_{H_{1}}\right] \ldots\left[\underline{P}_{H_{q}}, \bar{P}_{H_{q}}\right]\right]
$$

then

$$
\begin{aligned}
M_{X} & =\left[m(\emptyset) m\left(A_{1}\right) \ldots m\left(A_{i}\right) \ldots m\left(A_{2^{q}-1}\right)\right] \\
& =\left[\underline{P}_{\emptyset} \underline{P}_{A_{1}} \ldots \sum_{B \mid B \subseteq A_{i}}(-1)^{\left|A_{i}\right|-|B|} \underline{P}_{B} \ldots\right]
\end{aligned}
$$

with $A_{i} \in 2^{\Omega}$.

As argued by Smets [54], the knowledge of measures $\operatorname{Bel}\left(A_{i}\right)$ and $\operatorname{Pls}\left(A_{i}\right)$ is equal to the knowledge of the basic mass assignment on the frame of discernment.

Nevertheless, as usually defined in works with interval valued probabilities [11], two conditions should be considered when defining the probability interval distribution:

$$
\sum_{i=1}^{q} \underline{P_{i}} \leq 1
$$

and

$$
\sum_{i=1}^{q} \overline{P_{i}} \geq 1
$$

\section{EVIDENTIAL NETWORKS}

In the modeling of complex systems for the analysis of their reliability or their performances, the variables which represent the system, its components, its function or the events of the system are related to each other. These relations can be represented by conditional dependencies. In this section, we propose to define an evidential network in order to represent the conditional dependencies between variables in a description space integrating uncertainty as belief masses in the meaning of the Dempster-Shafer theory.

The proposed evidential networks are directed acyclic graphs which represent uncertain knowledge as a random and epistemic ways [22]. An evidential network is defined as a couple: $G=((N, A), M)$, where $(N, A)$ represents the graph with $N$ the set of nodes, $A$ the set of edges and, $M$ represents the set of belief masses associated to each node. When a node is not a root node i.e. when it has got parents' nodes, its 
belief mass distribution is defined by a conditional belief mass table quantifying the relation between the node and its parents. When a node is a root, an a priori belief mass table is defined.

A discrete random variable $X$ is represented by a node $X \in N$ with its frame of discernment $\Omega$ constituted by $q$ mutually exhaustive and exclusive hypotheses (cf. eq.1). The vector $M(X)$, also called $M_{X}$, is the belief mass distribution over the $2^{q}$ focal sets $A_{i}^{X} . M(X)$ is defined by the following equation:

$$
\begin{array}{r}
M(X)=\left[m(X \subseteq \emptyset) m\left(X \subseteq A_{1}^{X}\right) \ldots m\left(X \subseteq A_{i}^{X}\right)\right. \\
\left.\ldots m\left(X \subseteq A_{2^{q}-1}^{X}\right)\right]
\end{array}
$$

with $m\left(X \subseteq A_{i}^{X}\right) \geq 0$ and $\sum_{A_{i}^{X} \mid A_{i}^{X} \in 2^{\Omega}} m\left(X \subseteq A_{i}^{X}\right)=1$, where $m\left(X \subseteq A_{i}^{X}\right)$ is the belief that variable $X$ verify the hypotheses of focal element $A_{i}^{X}$.

When a node is a child node, $M$ is represented by its own conditional belief mass table. Each conditional belief mass table defines the relation between the belief masses on the frame of discernment of the variable of each parents nodes and the belief masses of the frame of discernment of the child node. Figure 2 shows two nodes $X$ and $Y$ defined with the frame of discernment $2^{\Omega_{X}}:\left\{\emptyset, A_{1}^{X}, \ldots, A_{M}^{X}\right\}, 2^{\Omega_{Y}}:\left\{\emptyset, A_{1}^{Y}, \ldots A_{K}^{Y}\right\}$ and lies to a node $Z$ with its own frame of discernment $2^{\Omega_{Z}}:\left\{\emptyset, A_{1}^{Z}, \ldots A_{L}^{Z}\right\}$. The conditional belief mass table of $Z$ is defined by conditional belief masses $M(Z \mid X, Y)$ for each hypothesis $A_{i}^{Z}$ knowing the focal sets of its parents $X$ and $Y$. For a root node, i.e. without parent, the belief masse table is a vector representing the a priori belief mass distribution defining the amount of belief that a variable verifies the hypotheses of the frame of discernment.

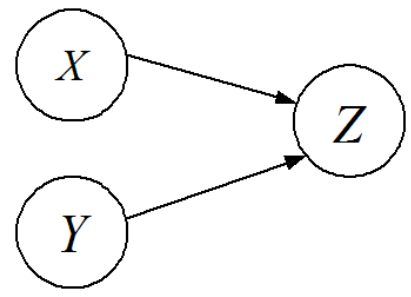

Fig. 2. Elementary network: 2 parents, 1 child

To compute the marginal belief mass distributions of each node, we use inference algorithms. The exact inference is carried out by the algorithm proposed by Jensen based on the construction of a junction tree [55, pp. 76]. This algorithm updates the marginal belief mass distributions on each node according to the evidence representing the knowledge introduced into the evidential network. The computation mechanism is based on the Bayes theorem, which is extended to the representation of uncertain information according to the framework of Dempster-Shafer theory (eq.20).

Specific evidence (Hard evidence) is modeled by a mass of 1 on one of the focal elements of the frame of discernment. Non-specific evidence (Soft evidence) corresponds to a mass distribution on the focal elements of the frame of discernment.

\section{A. Belief and plausibility measures}

To compute belief and plausibility measures in an evidential network, it is necessary to apply 7 and 8 . When an evidential network is implemented in a tool, the exact inference algorithm allows us to compute $\mathrm{Bel}$ and $\mathrm{Pls}$ measures. These measures cannot be computed in the same node because they are non additive measures. Simon and Weber [22] proposed to compute each measures on a focal element of a variable by two particular nodes (cf. figure 3). The node dedicated to compute

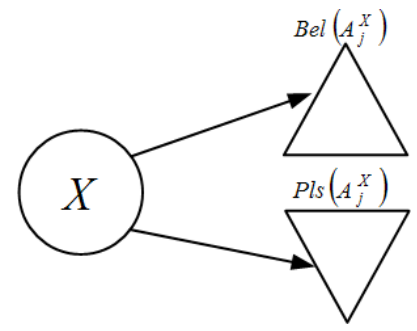

Fig. 3. Nodes to compute $\mathrm{Bel}$ and $\mathrm{Pls}$ measures.

$\operatorname{Bel}\left(A_{j}^{X}\right)$ is described by two hypotheses Believe and Doubt according to the conditional belief mass table given on table I.

TABLE I

CONDitional BELIEF MASS TABLE OF NODE $\operatorname{Bel}\left(A_{j}^{X}\right)$

\begin{tabular}{|c||c|c|}
\hline \multicolumn{1}{|c||}{$A_{i}^{X} \in 2^{\Omega_{X}}$} & Believe & Doubt \\
\hline \hline$\ldots$ & $\left\{\begin{array}{l}\text { A } \begin{array}{l}\text { if } A_{i}^{X} \subseteq A_{j}^{X} ; \\
\text { else } \\
A_{i}^{X}\end{array} \\
\ldots\end{array}\right.$ & $\begin{cases}0 & \begin{array}{l}\text { if } A_{i}^{X} \subseteq A_{j}^{X} \\
\text { else } \\
1\end{array} \\
\ldots\end{cases}$ \\
\hline
\end{tabular}

The node dedicated to compute $P l s\left(A_{j}^{X}\right)$ is described by hypotheses Plausibility et Disbelief according to table II.

TABLE II

Conditional Belief MASS tABle of nOde $P l s\left(A_{j}^{X}\right)$

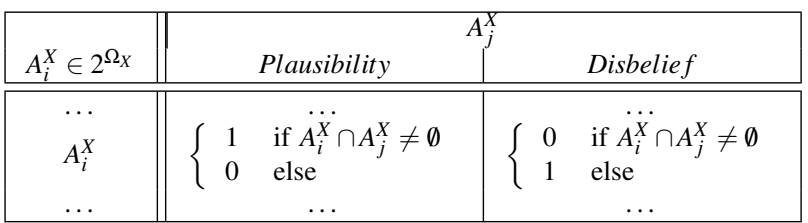

The structure of these nodes is generic. It is useful for the computation of belief and plausibility measures of each node of the network and for each hypothesis. Moreover, taking into account the bounding property (cf. 9), these nodes allow the definition of a p-box on any hypothesis of a studied variable.

\section{B. Integration of utility functions in evidential networks}

In some problems, a measure of satisfaction of a need or the achievement of a service is required. The concept of utility allows this measurement. Thus, for the evaluation of performances using the evidential networks, it is necessary to introduce the concept of utility.

The utility function translates the behavior of a decision maker according to risk. It is a function, which the decision maker must define for each decision. It is ordinal if the decision maker preferably expresses a relation between the decisions or cardinal if a mathematical function (for example 


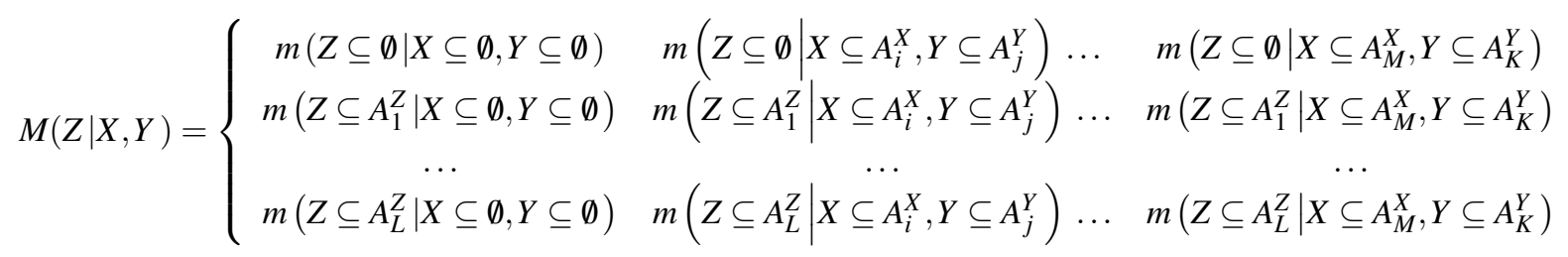

linear) is used to formalize the preference quantitatively. Thus, the goal is to build function $U$ from the decision space to $\mathbb{R}^{+}$such as $U(A)>U(B)$ implies that decision $A$ is preferred to decision $B$. More generally, the decision can take account of several parameters, it is a function of $\mathbb{R}^{n}$ in $\mathbb{R}$. Thus, the higher the value of the function $U$ for a decision is, the more appreciated is this decision. The function $U: \mathbb{R}^{n} \mapsto \mathbb{R}$ represents the preference $\succeq$ of the decision maker if and only if whatever $Z=\left(z_{1}, z_{2}, \ldots, z_{n}\right)$ and $Z^{\prime}=\left(z_{1}^{\prime}, z_{2}^{\prime}, \ldots, z_{n}^{\prime}\right)$, we have :

$$
U\left(z_{1}, z_{2}, \ldots, z_{n}\right) \geq U\left(z_{1}^{\prime}, z_{2}^{\prime}, \ldots, z_{n}^{\prime}\right) \Leftrightarrow Z \succeq Z^{\prime}
$$

If we associate consequences $X:\left\{H_{k}^{1}, \ldots, H_{k}^{j}, \ldots, H_{k}^{n}\right\}$ to each decision $S_{d}:\left\{s_{1}^{d}, \ldots, s_{k}^{d}, \ldots, s_{K}^{d}\right\}$ that can take $K$ 's states and probabilities $P_{k}:\left\{p_{k}^{1}, \ldots, p_{k}^{j}, \ldots, p_{k}^{n}\right\}$ to each consequence, the decision-maker choose the decision state $s_{k}^{d}$ that maximizes the expected utility $E U\left(s_{k}^{d}\right)$ computed by the following relation [56] :

$$
E U\left(s_{k}^{d}\right)=\sum_{j=1}^{n} p_{k}^{j} \cdot u\left(H_{k}^{j}\right)
$$

with

$$
p_{k}^{j}: P\left(X=H_{k}^{j} \mid S_{d}=S_{k}^{d}\right)
$$

and $u\left(H_{k}^{j}\right)$ is the utility of $H_{k}^{j}$.

Utility functions can be used in decision networks as we can read in [57], [58] and combines perfectly with Bayesian networks as Jensen shows in [55, p. 134] (cf. figure 4). To compute relation 22 , the weights $u\left(H_{k}^{j}\right)$ should be defined in an utility node associated to a node of the network, i.e. with a random variable.

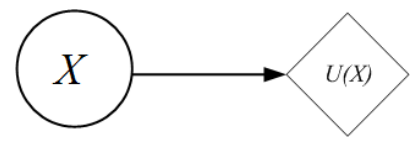

Fig. 4. Utility node of $X$

The use of the utility function in evidential networks rests on the same principle. However, the probabilities $P_{k}$ are replaced by belief and plausibility measures from which we deduce the associated belief masses $M_{k}:\left\{m_{k}^{1}, \ldots, m_{k}^{j}, \ldots, m_{k}^{2^{n}}\right\}$. Indeed, the nodes of the evidential network on which the utility is computed contains only belief masses. However, the utility was defined on specific hypothesis in the case of probabilities. In the case of belief masses, it is necessary to define a utility associated to the focal sets of the frame of discernment related to the node representing the studied variable, while respecting the logic of attribution of the utilities in the probabilistic case [59]. Thus, the relation 22 is divided into two equations related to the belief measure or lower probability and the plausibility measure or upper probability to define the lower expected utility (cf. 23) and upper expected utility (cf. 24).

$$
\underline{E U}\left(s_{k}^{d}\right)=\sum_{j=1}^{2^{n}} m_{k}^{j} \cdot \underline{u}\left(A_{k}^{j}\right)
$$

and

$$
\overline{E U}\left(s_{k}^{d}\right)=\sum_{j=1}^{2^{n}} m_{k}^{j} \cdot \bar{u}\left(A_{k}^{j}\right)
$$

If we consider non-specific hypothesis $A_{j}=\left\{H_{k}^{i}, H_{k}^{l}\right\}$, meaning that hypothesis $H_{k}^{i}$ or $H_{k}^{l}$ is verified knowing that $H_{k}^{i}$ and $H_{k}^{l}$ are mutually exclusive, the corresponding utility $u\left(A_{j}=\left\{H_{k}^{i}, H_{k}^{l}\right\}\right)$ must be given by the utilities $u\left(H_{k}^{i}\right)$ and $u\left(H_{k}^{l}\right)$. Thus, in the computation of lower expected utility $\underline{u}\{$.$\} ,$ which is the pessimistic case, we propose to use the least preferable utility among utilities $u\left(H_{k}^{i}\right)$ and $u\left(H_{k}^{l}\right)$ by checking the dominance properties between the hypothesis [59, p.110]. For higher expected utility $\bar{u}\{$.$\} , the most preferable utility is$ selected. Equations 23 and 24 becomes:

$$
\underline{E U}\left(s_{k}^{d}\right)=\sum_{j=1}^{2^{n}} m_{k}^{j} \cdot \underline{u}\left(A_{k}^{j}\right)
$$

with

$$
\underline{u}\left(A_{k}^{j}=\left\{H_{k}^{i}, H_{k}^{l}\right\}\right)=\min \left(u\left(H_{k}^{i}\right), u\left(H_{k}^{l}\right)\right)
$$

and

$$
\overline{E U}\left(s_{k}^{d}\right)=\sum_{j=1}^{2^{n}} m_{k}^{j} \cdot \bar{u}\left(A_{k}^{j}\right)
$$

with

$$
\bar{u}\left(A_{k}^{j}=\left\{H_{k}^{i}, H_{k}^{l}\right\}\right)=\max \left(u\left(H_{k}^{i}\right), u\left(H_{k}^{l}\right)\right)
$$

The main requirement to define utilities is to warrant $\overline{E U}\left(s_{k}^{d}\right) \geq \underline{E U}\left(s_{k}^{d}\right)$ and can be translated into utilities as follows:

$$
u\left(H_{k}^{i}\right) \geq \bar{u}\left(A_{k}^{j}=\left\{H_{k}^{i}, H_{k}^{l}\right\}\right) \geq \underline{u}\left(A_{k}^{j}=\left\{H_{k}^{i}, H_{k}^{l}\right\}\right) \geq u\left(H_{k}^{l}\right)
$$

We define the utility in evidential networks from the probabilistic case where all $u\left(H_{k}^{j}\right)$ are known. Thus, all $u\left(A_{k}^{i}\right)$ are deduced. On this basis, the utilities in eq.25 and 27 are easily obtained. Nevertheless, experts may want to change them while they respect eq.29. 


\section{Discussion}

The evidential networks proposed here are based on the exact inference algorithm and junction tree as defined by Jensen in [55]. The imprecision on probabilities is coded by Dempster Shafer structures (frame of discernment) introduced on each root nodes. The focal elements that combine elementary hypothesis of the probability framework, quantitatively support the imprecision on elementary probabilities.

The conditional belief mass tables explain the relation between variables and also the imprecision. The computation of inference is made with the total probability theorem or the Bayes theorem extended to belief masses in the same way as proposed by Jensen. Thus, the computational complexity in Evidential networks is NP-hard as Cooper defined it for Bayesian networks [60].

At last, believe and plausibility measures, or superior and inferior bounds, are computed by particular conditional mass tables in two separated nodes in order to respect the additivity axiom inherent to the inference algorithm used.

One main advantage to code Dempster Shafer structure into evidential network is to directly tackle imprecision without choosing probability laws for elementary probabilities and, without using Monte Carlo simulations. In the latter case, uniform laws were usually chosen and a normal law is obtained for the top event considering the central limit theorem. As mentioned by Ferson [53], the central limit theorem is widely abused as a justification for a normal or lognormal distribution shape and usually we didn't know the initial distributions. Another advantage of evidential networks is that belief masses are additives and found a good correspondence with the inference algorithm.

\section{RELIABILITY MODELING BY EVIDENTIAL NETWORKS}

As Rakowsky argues [48], the community of safety and reliability engineers discovered the Dempster-Shafer theory through the work of Guth [45] at the beginning of the Nineties. Guth proposes to compute the probist reliability [15] of a system by a fault tree containing three hypotheses on the state of the components or the system. Actually, as argued by Simon and Weber [22], Guth extends the frame of discernment corresponding to the hypotheses of the probist reliability to the frame of discernment of the Dempster-Shafer theory:

- Probabilistic hypothesis: the system functioning is completely described by probability measures.

- Binary state hypothesis: the system can have only two operating conditions, the state of failure $\{$ Down $\}$ and the normal operating state $\{U p\}$.

Thus, the probabilistic frame of discernment $(\Omega=$ $\{\{U p\},\{$ Down $\}\})$ becomes a Dempster-Shafer structure $\left(2^{\Omega}=\{\emptyset,\{U p\},\{\right.$ Down $\},\{U p$, Down $\left.\}\}\right)$ which can be reduced to the three hypothesis:

- $m\{U p\}$ : belief mass to be in operating condition,

- $m\{$ Down $\}$ : belief mass to be in fail condition,

- $m\{U p$, Down $\}$ : belief mass to be exclusively in one of the previous conditions without distinguishing exactly which.

Under the assumptions of probist reliability, the studied components as well as the system can be only in one of the two operating conditions. This is a closed world problem [43] and the hypothesis $\emptyset$ does not carry any belief mass. In the analysis of reliability or risk integrating human factors, it can be interesting to assign a belief mass to $\emptyset$ to characterize the lack of completeness of assumptions (open world) on which carries the analysis rather than to introduce a safety coefficient or a margin of probability on the global result in order to take account the possible missed scenarios in the analysis. In this study, we consider the problem of reliability analysis or the performance analysis of systems as a problem of closed world $(m(\emptyset)=0)$.

To model the reliability of systems by evidential networks, we transpose the approach suggested by Bobbio et al. [61], [62] with Bayesian networks to evidential networks. The goal is to convert a fault tree into an equivalent network with the hypothesis suggested by Guth [45].

\section{A. Probist modeling}

A fault tree describes the propagation process of a failure within the functional structure of a system. The reliability of the modeled system follows the assumptions of independence of the events and of coherence of the systems [63]. The reliability is described by 'AND', 'OR', ' $k$-out-of- $n$ ':G gate combining the elementary events. To integrate the frame of discernment of the Dempster-Shafer theory, the evidential network must model the truth tables of 'AND' gate (cf. table III) and 'OR' gate (cf. table IV) [45] by tables of conditional belief mass [22].

TABLE III

TRUth table OF A 'AND' Gate

\begin{tabular}{|c||c|c|c|}
\hline AND & $\{$ Up $\}$ & $\{$ Down $\}$ & $\{$ Up,Down $\}$ \\
\hline \hline$\{U p\}$ & $\{$ Up $\}$ & $\{$ Down $\}$ & $\{$ Up,Down $\}$ \\
$\{$ Down $\}$ & $\{$ Down $\}$ & $\{$ Down $\}$ & $\{$ Down $\}$ \\
$\{$ Up,Down $\}$ & $\{$ Up,Down $\}$ & $\{$ Down $\}$ & $\{$ Up,Down $\}$ \\
\hline
\end{tabular}

TABLE IV

TRUTH TABLE OF A 'OR' GATE

\begin{tabular}{|c||c|c|c|}
\hline OR & $\{$ Up $\}$ & $\{$ Down $\}$ & Up,Down \\
\hline \hline$\{$ Up $\}$ & $\{$ Up $\}$ & $\{$ Up $\}$ & $\{$ Up $\}$ \\
$\{$ Down $\}$ & $\{$ Up $\}$ & $\{$ Down $\}$ & $\{$ Up,Down $\}$ \\
$\{$ Up,Down $\}$ & $\{$ Up $\}$ & $\{$ Up,Down $\}$ & $\{$ Up, Down $\}$ \\
\hline
\end{tabular}

The conditional belief mass table representing a 'AND' gate is defined by table V. $E_{X}$ corresponds to the state of the component $X, E_{Y}$ to the state of component $Y$, and $E_{X}, E_{Y}$ are the inputs of the 'AND' gate. $E_{Z}$ corresponds to the output of the gate. The conditional belief mass table of a 'OR' gate is defined by table VI.

The conditional belief mass table can be adapted to gates with more inputs and also to $k$-out-of- $n: G$ gates (cf. table VII). In addition, the coefficients of the conditional belief mass table take their value in $\{0,1\}$ since it is a translation of the truth tables of logical gates. These coefficients can take different values from $\{0,1\}$ if the modeling of different behaviors is expected in particular when there is an uncertainty about the propagation of belief masses in the evidential network. 
TABLE V

CONDITIONAL BELIEF MASS TABLE OF A 'AND' GATE

\begin{tabular}{|c|c|c|c|c|}
\hline$E_{X}$ & $E_{Y}$ & $\{\mathrm{Up}\}$ & $\begin{array}{r}E_{Z} \\
\{\text { Down }\}\end{array}$ & $\{$ Up,Down $\}$ \\
\hline$\{\mathrm{Up}\}$ & $\{\mathrm{Up}\}$ & 1 & 0 & 0 \\
\hline$\{$ Down $\}$ & $\{\mathrm{Up}\}$ & 0 & 1 & 0 \\
\hline$\{$ Up,Down $\}$ & $\{\mathrm{Up}\}$ & 0 & 0 & 1 \\
\hline$\{\mathrm{Up}\}$ & $\{$ Down & 0 & 1 & 0 \\
\hline$\{$ Down & $\{$ Down & 0 & 1 & 0 \\
\hline$\{\mathrm{Up}$, Down $\}$ & $\{$ Down $\}$ & 0 & 1 & 0 \\
\hline$\{\mathrm{Up}\}$ & $\{$ Up,Down $\}$ & 0 & 0 & 1 \\
\hline$\{$ Down $\}$ & $\{$ Up,Down $\}$ & 0 & 1 & 0 \\
\hline$\{$ Up,Down $\}$ & $\{$ Up,Down $\}$ & 0 & 0 & 1 \\
\hline
\end{tabular}

TABLE VI

CONDITIONAL BELIEF MASS TABLE OF A 'OR' GATE

\begin{tabular}{|c|c||c|c|c|}
\hline & & \multicolumn{3}{|c|}{$E_{Z}$} \\
$E_{X}$ & $E_{Y}$ & $\{\mathrm{Up}\}$ & $\{$ Down & $\{\mathrm{Up}$, Down $\}$ \\
\hline \hline$\{\mathrm{Up}\}$ & $\{\mathrm{Up}\}$ & 1 & 0 & 0 \\
$\{$ Down & $\{\mathrm{Up}\}$ & 1 & 0 & 0 \\
$\{\mathrm{Up}$, Down $\}$ & $\{\mathrm{Up}\}$ & 1 & 0 & 0 \\
$\{\mathrm{Up}\}$ & $\{$ Down & 1 & 0 & 0 \\
$\{$ Down & $\{$ Down & 0 & 1 & 0 \\
$\{\mathrm{Up}$, Down & $\{$ Down & 0 & 0 & 1 \\
$\{\mathrm{Up}\}$ & $\{\mathrm{Up}$, Down & 1 & 0 & 0 \\
$\{$ Down & $\{\mathrm{Up}$, Down & 0 & 0 & 1 \\
$\{\mathrm{Up}$, Down $\}$ & $\{\mathrm{Up}$, Down & 0 & 0 & 1 \\
\hline
\end{tabular}

1) Plausibility and belief measures: To define the imprecise probist reliability of a system, it is necessary to compute the p-box on the hypothesis $\{U p\}$ of the belief mass distribution of the system using adapted nodes as it was defined in section III-A. The conditional belief masses of belief measure node $\operatorname{Bel}(\{U p\})$ is given by table VIII and those of plausibility measure is given by table IX.

TABLE VIII

CONDITIONAL BELIEF MASS TABLE OF NODE $\operatorname{Bel}(\{U p\})$

\begin{tabular}{|c||c|c|}
\hline & \multicolumn{2}{|c|}{$\operatorname{Bel}(\{U p\})$} \\
& Believe & Doubt \\
\hline \hline$\{$ Up $\}$ & 1 & 0 \\
$\{$ Down $\}$ & 0 & 1 \\
$\{$ Up,Down $\}$ & 0 & 1 \\
\hline
\end{tabular}

TABLE IX

CONDITIONAL BELIEF MASS TABLE OF NODE $P l s(\{U p\})$

\begin{tabular}{|c||c|c|}
\hline & \multicolumn{2}{|c|}{$P l s(\{U p\})$} \\
& Plausibility & Disbelief \\
\hline \hline$\{$ Up $\}$ & 1 & 0 \\
$\{$ Down $\}$ & 0 & 1 \\
$\{$ Up,Down $\}$ & 1 & 0 \\
\hline
\end{tabular}

2) Simplified Development of conditional belief mass table: The growth of the size of set of states $\Omega$ immediately implies the growth of the size of the frame of discernment requiring a great effort of modeling. Thus, for a component or system in two states, $|\Omega|=2$, the frame of discernment comprises $\left|2^{\Omega}\right|=4$ hypotheses and 4 a priori belief masses to define. If we consider that $\emptyset$ is not taken into account in the study, the cardinal of the useful frame of discernment is equal to 3 . When 3 states are considered, $\left|2^{\Omega}\right|=8$ and even if $\emptyset$ is not used, $7 a$ priori belief masses are to be defined. The growth of the size of the frame of discernment influences directly the gates modeled by the conditional belief mass tables. Indeed, a 'AND' gate with 2 inputs and 1 output with 3 possible assumptions each forces the definition of $7^{3}$ conditional masses.

This growth of the number of a priori and conditional belief masses is a major drawback for easy use of the DempsterShafer theory and has been often used to reject its use. Within the studies done in this article, the effort required for the definition of the conditional belief mass tables can be largely reduced by the use of the De Morgan's laws. Let us consider the conditional belief mass table a 'AND' gate with two inputs $X, Y$ and 1 output $Z$ (cf. table V). The definition of the third line of this table is done from the first 2 lines. Let us recall that according to probist reliability, $\{U p, D o w n\}$ means that the component is either in the state $U p$, or in the state Down. By no means, a component can be in both states simultaneously (exclusive assumption). Thus, $\{U p, D o w n\}$ is interpreted as $\{U p\} \oplus\{$ Down $\}$ where $\oplus$ is the or exclusive logical operator. The equation of the third line of table $\mathrm{V}$ is written $\{U p, D o w n\} .\{U p\}$ and becomes :

$$
\begin{aligned}
\{U p \oplus \text { Down }\} .\{U p\} & =(\{U p\} \cdot\{U p\}) \oplus(\{U p\} .\{\text { Down }\}) \\
& =\{U p\} \oplus\{\text { Down }\} \\
& =\{U p, \text { Down }\}
\end{aligned}
$$

Consequently, for a 'AND' gate with 2 inputs, only 8 conditional belief masses corresponding to the combinations of specific states of a usual 'AND' gate are to be defined among the 27 of the table. The other conditional belief masses are automatically deduced by the De Morgan's laws.

As Weber claims with Bayesian networks [7], the knowledge of the components or system operating modes is not necessarily precise and can be uncertain. This can also concerns evidential networks and the conditional belief mass table. The conditional belief masses do not take their values from $\{0,1\}$ but in $[0,1]$. Thus, we have more flexibility to describe the influence between the operating modes. In this last case, there is no possible reduction of the analysis effort by the use of the De Morgan's laws to define the conditional belief masses. The analyst must define each mass according to effective relations between the operating modes. However, these conditional belief masses can be estimated from databases.

3) Imprecise reliability parameters: Generic databases of reliability are often used to provide the failure rates of the components of systems in particular for the safety instrumented systems [64]-[67]. Scalar values of failure rates are generally used to estimate the failure probabilities of components. However, the data acquisition of reliability and the use of this data to estimate the failure rates of other components of the same type introduce uncertainties. According to [68], the data of reliability collected for a component can change with a range of 3 or 4, and sometimes a range of 10. In [63], [69], the authors propose to use coefficients of influence to take account the real conditions of use of the generic values of failure rates suggested by the databases of reliability. Some databases of reliability [70]-[72] provide the lower and upper limits, the average or median values and the error factors of the component failure rate. 
When we work with lower and upper bounds for the constant failure rates of a component $X$ coming from databases ( $\left[\underline{\lambda}_{X}, \bar{\lambda}_{X}\right]$ ), it is easy to define the bounds of the component reliability at a time of mission $T_{i}$ given using the following equation:

$$
\left[\underline{P}_{X}, \bar{P}_{X}\right]=1-\exp \left(\left[\underline{\lambda}_{X}, \bar{\lambda}_{X}\right] . T_{i}\right)
$$

The definition of associated belief mass distribution is easily determined by 16 . In the case of probist reliability, we write:

$$
\begin{aligned}
M_{X}= & {[m(\{U p\} m(\{\text { Down }\} m(\{U p, \text { Down }\}]} \\
= & {\left[\underline{P}_{X}(\{U p\})\left(1-\bar{P}_{X}(\{U p\})\right)\right.} \\
& \left.\left(\bar{P}_{X}(\{U p\})-\underline{P}_{X}(\{U p\})\right)\right]
\end{aligned}
$$

\section{B. Multistate systems}

In many cases, the system as well as its components can function in various states or operating modes characterized by various levels of performance. Such systems refer to multistate systems [73]. Applied to multistate systems, a reliability analysis allows computing a measure of the capacity of a system to provide a necessary level of performance according to its level of degradation. Multistate systems can also be subjected to undetected failures, which lead to the complete failure of the system or its subsystems.

At the same time, the binary state assumption of components' operating modes can be removed because it does not suitably represent the degradation of components' operating mode of systems [15], [74]. Various approaches allow handling the degradations of components. Some authors propose to deal with this problem within the framework of profust reliability [74]-[76]:

- Probability assumption: the operating condition of the system is completely characterized by probability measures.

- Fuzzy state assumption: the operating condition of the system is characterized by two fuzzy states allowing a gradual transition between the normal operating condition to the failure state [77].

A second solution is to propose a description of the operating condition of the components and of the system by several states [78]. The accuracy of the description is more or less closed to the real state of the degradation and operating modes of the components or of the system.

1) Modeling of the reliability of multistate systems by evidential networks: To analyze the reliability or the performance of multistate systems, it is necessary to know all the operating modes of the studied system and of its components. The operating mode of each component is supposed to be independent of the operating mode of the other components. Moreover, the system is supposed to be coherent.

Let us consider a system with $K+1$ operating modes $S=\left\{s_{0}, \ldots, s_{K}\right\}$ and levels of performance $U=\left\{u_{0}, \ldots, u_{K}\right\}$ expressed like utility. Each level of performance $u_{i}$ corresponds to an operating mode $s_{i}$ of the system. Operating mode $s_{K}$ corresponds to normal operation and is associated to the maximum level of performance $u_{K}$. Mode $s_{0}$ corresponds to a total failure of the system, which induces a minimum level of performance $u_{0}$.

The system is composed of $n$ components $C_{i}$ having each one $K_{C_{i}}+1$ operating modes $S_{C_{i}}=\left\{s_{C_{i} 0}, \ldots, s_{C_{i} K_{C_{i}}}\right\}$. Operating mode $s_{C_{i} K_{C_{i}}}$ is the normal operating mode of the component and mode $s_{C_{i} 0}$ corresponds to the state of total failure of the component. The operating condition of the system depends on the operating condition of its components. Thus, the reliability of the system is expressed as the following structure function $\phi$ :

$$
S=\phi\left(S_{C_{1}}, \ldots, S_{C_{n}}\right): S_{C_{i}}^{n} \rightarrow S
$$

with

$$
S_{C_{i}}^{n}=\left\{s_{C_{1} 0}, \ldots, s_{C_{1} K_{C_{1}}}\right\} \times \ldots \times\left\{s_{C_{n} 0}, \ldots, s_{C_{n} K_{C_{n}}}\right\}
$$

where $S_{C_{i}}^{n}$ is the space of all possible combinations of components' operating modes and $S$ is the space of the system operating modes. At any time, the operating mode of a multistate system can be described by a random variable $Y=\left\{y_{0}, \ldots, y_{k}, \ldots, y_{K}\right\}$. The operating mode of each component can be also described a random variable $X_{C_{i}}=$ $\left\{x_{C_{i 0}}, \ldots, x_{C_{i K_{C}}}\right\}$. Then, we can write $Y$ as a combination of the random variables $X_{C_{i}}$ according to the structure function $\phi$.

$$
Y=\phi\left(X_{C_{i}}\right)
$$

At every time, the level of performance of the system is computed by the expected utility according to the following equation:

$$
U=\sum_{k=0}^{K} y_{k} \cdot u_{k}
$$

As in Bayesian networks [7], to take into account the multiple operating modes of a system and of its components in the reliability analysis by an evidential networks is easy. It consists in defining the a priori belief mass tables and the conditional belief mass tables. The qualitative aspect of the network, i.e. the graph, models the propagation mechanism of the influence of the operating modes, i.e. it models the structure function $\phi$. The interest of using evidential networks is to represent the structure function $\phi$ synthetically in a factorized way while taking into account imprecision and uncertainties.

We can also claim that the definition of the conditional belief mass tables for multistate systems is more tiresome because of the exponential growth of the combinations of operating modes. However, as we specified in section IV-A2, it is possible to reduce this stage, either by carrying out an estimation or by using the De Morgan's laws. The De Morgan's laws allow simplifying the definition of all nonspecific hypotheses from the relations between specific hypotheses.

2) Imprecise probabilities in multistate systems: In multistate systems, the probability for each component to be in one of its operating modes is given as a scalar value. If the provided probabilities are imprecise, these probabilities can be defined by intervals $\left[P_{i}^{X}, \overline{P_{i}^{X}}\right]$ where $X$ is the random variable characterizing the operating mode of an element. The 
translation of probability intervals to belief mass distribution is done as in the previous section by 16 or by the set of equations 7, 8 and 13 .

For instance, let us consider a system composed of components with three operating modes each $S=$ $\left\{s_{2}, s_{1}, s_{0}\right\}$ and three associated levels of performance $U=$ $\left\{u_{2}, u_{1}, u_{0}\right\}$ where $u_{2}$ is the most powerful level and $u_{0}$ the least. The frame of discernment is thus: $2^{\Omega}=$ $\left\{\left\{s_{2}\right\},\left\{s_{1}\right\},\left\{s_{0}\right\},\left\{s_{2}, s_{1}\right\},\left\{s_{2}, s_{0}\right\},\left\{s_{1}, s_{0}\right\},\left\{s_{2}, s_{1}, s_{0}\right\}\right\}$.

If we lay out probability intervals at one mission time $T_{i}$ for each defined mode:

$$
[P]=\left[\left[\underline{P}_{s_{2}}^{X}, \bar{P}_{s_{2}}^{X}\right],\left[\underline{P}_{s_{1}}^{X}, \bar{P}_{s_{1}}^{X}\right],\left[\underline{P}_{s_{0}}^{X}, \bar{P}_{s_{0}}^{X}\right]\right]
$$

Then, the a priori belief mass table are defined by:

$$
\begin{array}{r}
M=\left[m\left(\left\{s_{2}\right\}\right) m\left(\left\{s_{1}\right\}\right) m\left(\left\{s_{0}\right\}\right) m\left(\left\{s_{2}, s_{1}\right\}\right) m\left(\left\{s_{2}, s_{0}\right\}\right)\right. \\
\left.m\left(\left\{s_{1}, s_{0}\right\}\right) m\left(\left\{s_{2}, s_{1}, s_{0}\right\}\right)\right]
\end{array}
$$

According to 13, the following relations are obtained:

$$
\begin{aligned}
& m\left(\left\{s_{2}\right\}\right)=\operatorname{Bel}\left(\left\{s_{2}\right\}\right)=\underline{P}\left(\left\{s_{2}\right\}\right) \\
& m\left(\left\{s_{1}\right\}\right)=\operatorname{Bel}\left(\left\{s_{1}\right\}\right)=\underline{P}\left(\left\{s_{1}\right\}\right) \\
& m\left(\left\{s_{0}\right\}\right)=\operatorname{Bel}\left(\left\{s_{0}\right\}\right)=\underline{P}\left(\left\{s_{0}\right\}\right)
\end{aligned}
$$

Moreover:

$$
m\left(\left\{s_{2}, s_{1}\right\}\right)=-\operatorname{Bel}\left(\left\{s_{2}\right\}\right)-\operatorname{Bel}\left(\left\{s_{1}\right\}\right)+\operatorname{Bel}\left(\left\{s_{2}, s_{1}\right\}\right)
$$

and from 7 and 8 :

$$
\operatorname{Bel}\left(\left\{s_{2}, s_{1}\right\}\right)=1-\bar{P}\left(\left\{s_{0}\right\}\right)
$$

The same approach can be carried out to compute the probability interval on each focal element of the a priori belief mass table as specifies by 16 .

\section{STUdY CASE}

This section is dedicated to the analysis of the suggested evaluation method of system reliability. We study two systems of different complexity with on the one hand two operating conditions and on the other hand a multistate point of view. Two cases are distinguished according to whether the probabilities are precise or not. The inference in evidential networks is made by the algorithm of exact inference defined in Bayesialab(c). Evidential networks are directly modeled by using the graphic interface of this tool.

\section{A. Probist reliability of a 2-out-of-3:G system}

For the first example, we propose to simulate a 2-out-of$3: \mathrm{G}$ system. The goal is to show the capacity of evidential networks to compute the systems reliability. The evidential network of a 2-out-of-3:G system is defined in BayesialabC with the structure presented on figure 5 and the conditional belief mass table (TableauVII).

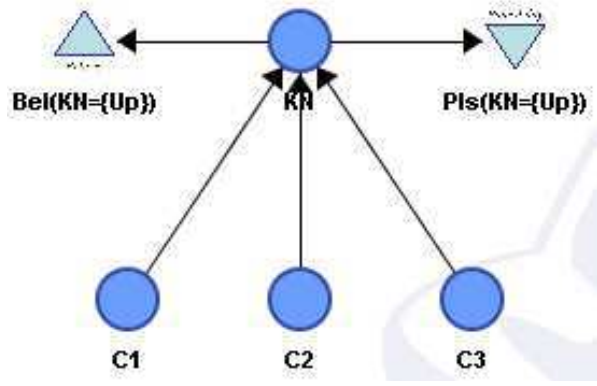

Fig. 5. Evidential network of a 2-out-of-3:G system

1) Precise probabilities: To simplify the example and without loss of generality, let us consider that each component has the same failure rate $\lambda$. The elementary events which lead the components from state $\{U p\}$ to state $\{$ Down $\}$ are independent. The system is homogeneous and no repair is considered. The system reliability $R\left(T_{i}\right)$ is given by the following equation:

$$
\begin{aligned}
& R\left(T_{i}\right)=\sum_{i=k}^{n} \mathrm{C}_{n}^{i} r\left(T_{i}\right)^{i}\left(1-r\left(T_{i}\right)\right)^{n-i} \\
& \text { avec } \mathrm{C}_{n}^{i}=\frac{n !}{i !(n-i) !}
\end{aligned}
$$

where $r\left(T_{i}\right)$ is the probability of each component to be in state $\{U p\}, T_{i}$ is the time of mission and $k$ the number of working components out of $n$.

Let us consider $\lambda=10^{-3} h^{-1}$ and $T_{i}=200 h$. The probability for each component $C_{i}$ to be in state $\{U p\}$ is given by: $P_{C_{i}}(\{U p\})=0.81873$. The a priori belief mass distribution of node $C_{i}$ is:

$$
\begin{array}{r}
M_{C_{i}}=[m(\{U p\})=0.8187 m(\{\text { Down }\})=0.18127 \\
m(\{U p, \text { Down }\})=0]
\end{array}
$$

Without imprecision on the value of $\lambda$, belief mass $m(\{U p$, Down $\})=0$ expresses that evidences are Bayesian and $\operatorname{Bel}\left(C_{i}=\{U p\}\right)=P\left(C_{i}=\{U p\}\right)=P l s\left(C_{i}=\{U p\}\right)$. So, the problem is completely Bayesian and follows 38. The system reliability is $R\left(T_{i}\right)=0.9133$. Figure 6 shows that the evidential network computes the exact value of the system reliability.

This simple example shows that the coding of a priori belief masses with Bayesian evidences in the evidential network gives the exact value of the system reliability. Thus, as claimed by Simon and Weber in [22], there is a complete equivalence between evidential networks and other probabilistic methods such as Bayesian networks in computation of system reliability.

2) Imprecise probabilities: Now, let us consider an imprecise failure rate of the components as an interval: $[\underline{\lambda}, \bar{\lambda}]=$ $\left[0.9 e^{-3}, 1.1 e^{-3}\right]$. The a priori belief mass distribution defining each component state at mission time $T_{i}=200 \mathrm{~h}$ is obtained by $7,8,13$ and 31 :

$$
\begin{aligned}
M_{C}=[m(\{U p\})=0.80252 m(\{\text { Down }\}) & =0.16473 \\
m(\{U p, \text { Down }\}) & =0.03275]
\end{aligned}
$$

The imprecise value of $\lambda$ induces a belief mass $m(\{U p$, Down $\})>0$ expressing the doubt about the 


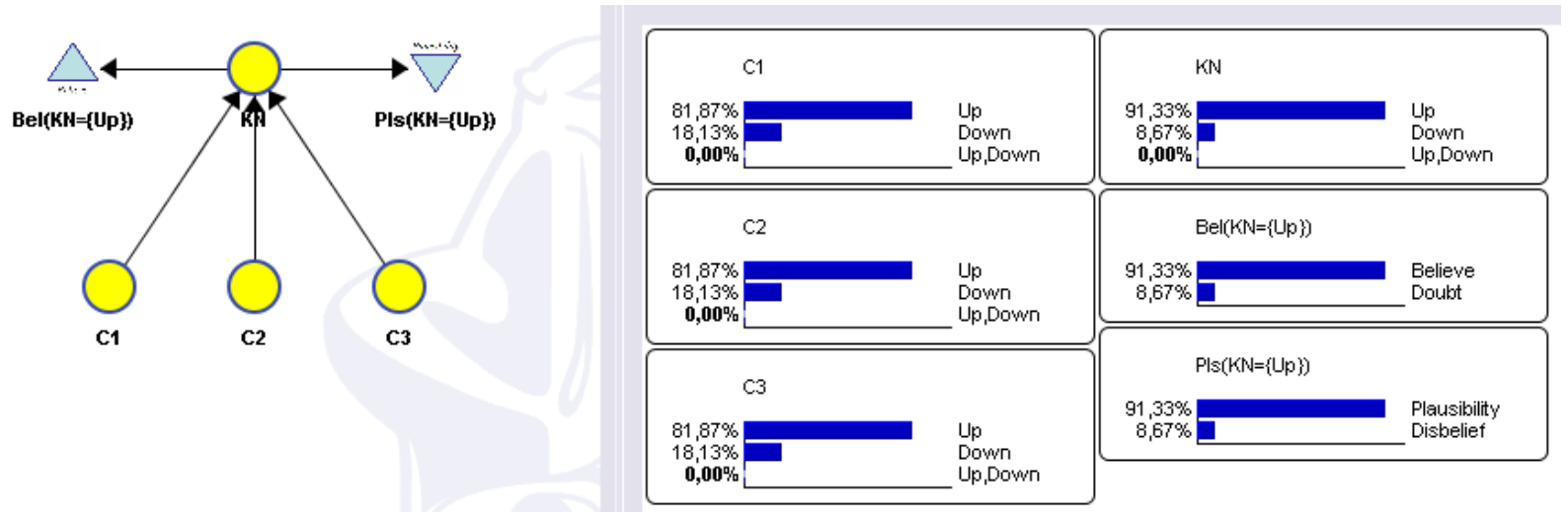

Fig. 6. Reliability of a 2-out-of-3:G gate with precise probabilities

knowledge of the exact value of $\lambda$. It translates a non strict Bayesian frame and $\operatorname{Bel}\left(C_{i}=\{U p\}\right)<P\left(C_{i}=\{U p\}\right)<$ $\operatorname{Pls}\left(C_{i}=\{U p\}\right)$. Figure 7 shows the result obtained by the evidential network.

The system reliability is obtained by the probability of the system to be in operating condition $(\{U p\})$ at time $T_{i}$. It is between $\left[\underline{R}\left(T_{i}\right), \bar{R}\left(T_{i}\right)\right]=[0.8984,0.9275]$. Compared to the result obtained in the previous section, we notice that the bounding property (cf. 9) is verified :

$$
\begin{array}{r}
\operatorname{Bel}(\{U p\})=0.8984<P(\{U p\})=0.9133 \\
P(\{U p\})<P l s(\{U p\})=0.9275
\end{array}
$$

with

$$
\operatorname{Bel}(\{U p\})=\underline{P}(\{U p\}) \text { et } P l s(\{U p\})=\bar{P}(\{U p\})
$$

\section{B. 2-out-of-3:G multistate system}

In order to show the mechanism of evidential networks for the study of multistate system performances, we study the 2-out-of-3:G system provided by Gopal [79]. The system is composed of 3 components with 3 operating modes each $S_{C_{i=1,2,3}}=\left\{s_{i 0}, s_{i 1}, s_{i 2}\right\}$. The system also has 3 operating modes $S=\left\{s_{0}, s_{1}, s_{2}\right\}$ associated to 3 levels of performance $U=\left\{u_{0}, u_{1}, u_{2}\right\}$. The structure function $\phi$ giving the relation between the components' operating modes and the system operating modes is given by the following table:

TABLE $X$

STRUCTURE FUNCTION OF A MULTISTATE 2-OUT-OF-3:G SYSTEM [79]

\begin{tabular}{|c|c|c||c||c|c|c||c|}
\hline$S_{C_{1}}$ & $S_{C_{2}}$ & $S_{C_{3}}$ & $\phi(S)$ & $S_{C_{1}}$ & $S_{C_{2}}$ & $S_{C_{3}}$ & $\phi(S)$ \\
\hline \hline 0 & 0 & 0 & $s_{0}$ & 1 & 1 & 2 & $s_{1}$ \\
0 & 0 & 1 & $s_{0}$ & 1 & 2 & 0 & $s_{1}$ \\
0 & 0 & 2 & $s_{0}$ & 1 & 2 & 1 & $s_{1}$ \\
0 & 1 & 0 & $s_{0}$ & 1 & 2 & 2 & $s_{2}$ \\
0 & 1 & 1 & $s_{1}$ & 2 & 0 & 0 & $s_{0}$ \\
0 & 1 & 2 & $s_{1}$ & 2 & 0 & 1 & $s_{1}$ \\
0 & 2 & 0 & $s_{0}$ & 2 & 0 & 2 & $s_{2}$ \\
0 & 2 & 1 & $s_{1}$ & 2 & 1 & 0 & $s_{1}$ \\
0 & 2 & 2 & $s_{2}$ & 2 & 1 & 1 & $s_{1}$ \\
1 & 0 & 0 & $s_{0}$ & 2 & 1 & 2 & $s_{2}$ \\
1 & 0 & 1 & $s_{1}$ & 2 & 2 & 0 & $s_{2}$ \\
1 & 0 & 2 & $s_{1}$ & 2 & 2 & 1 & $s_{2}$ \\
1 & 1 & 0 & $s_{1}$ & 2 & 2 & 2 & $s_{2}$ \\
1 & 1 & 1 & $s_{1}$ & & & & \\
\hline
\end{tabular}

The function defining the relation between system performance $U_{S}$ and the components operating modes is expressed as the expected utility (cf. 22) defined by the following relation:

$$
U_{S}=\sum_{s_{i}}\left(u_{i} \cdot y_{i}\right)=\sum_{s_{i}} u_{i} \cdot \phi\left(x_{C_{i}}\right)
$$

where $y_{i}$ is the probability of the system to be in operating mode $i, x_{C_{i}}$ is the probability of component $C_{i}$ to be in operating mode $i$ and $\phi$ the structure function given by table $\mathrm{X}$.

To compute the system performance by an evidential network, we translate the structure function suggested by Gopal (cf. table $\mathrm{X}$ ) by a conditional belief mass table in node $\mathrm{KN}$ on figure 8.

By adding an utility node $U s$, we compute the system performance according to 42 . The table of the corresponding utilities allows computing the performance (cf. table XI).

TABLE XI

TABLE OF UTILITIES

\begin{tabular}{|c|c|}
\hline Focal set & utility \\
\hline$s_{2}$ & 2 \\
$s_{1}$ & 1 \\
$s_{0}$ & 0 \\
\hline
\end{tabular}

1) Precise probabilities: According to Gopal [79], if the distribution of component operating modes is: $P_{S_{C_{i 1}}}=$ $(0.1,0.3,0.6), P_{S_{C_{i 2}}}=(0.3,0.5,0.2), P_{s_{C_{i 3}}}=(0.4,0.2,0.4)$ then the system performance is 0.822 units. We notice that the evidential network presented on figure 8 gives the same value of the expected utility with a priori belief masses according to the a priori probability distributions provided above.

2) Imprecise probabilities: Now let us consider the problem of imprecise probabilities on the component states. For example and without loss of generality, we will consider that the probabilities of the previous problem are given with an uncertainty \pm 0.05 . We must compute the performance as defined by Gopal [79]:

$$
U_{S}=\sum_{s_{i=1,2,3}} u_{i} . \phi\left(x_{C_{i}}\right)
$$




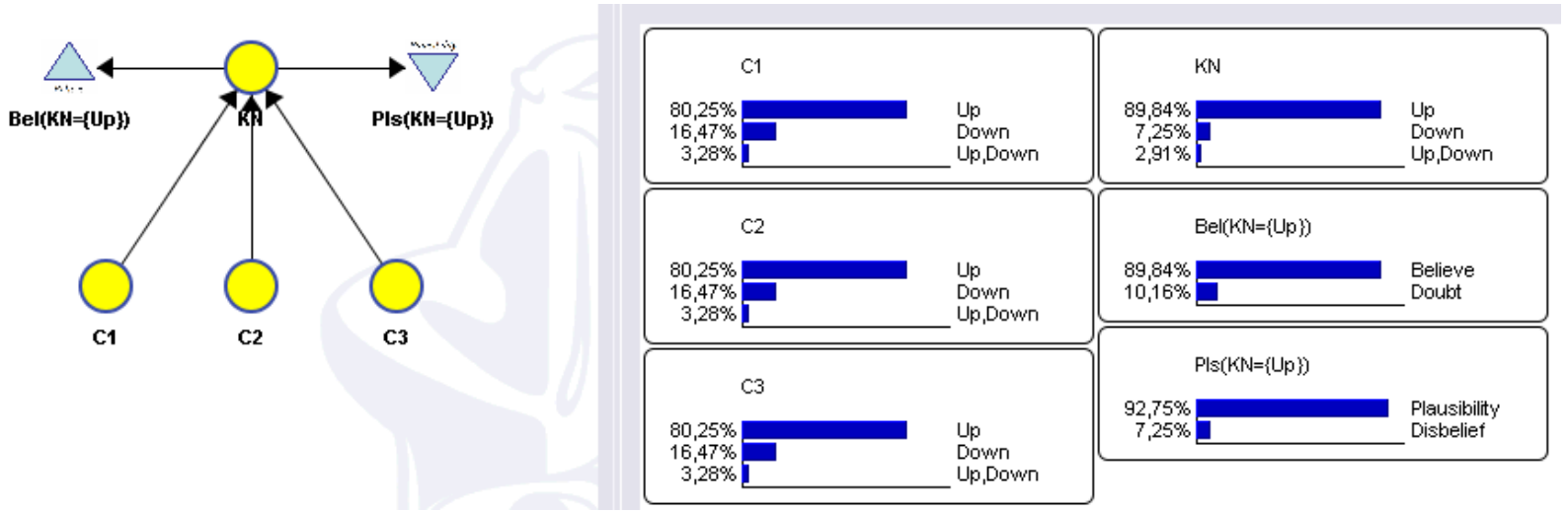

Fig. 7. Imprecise reliability of a '2-out-of-3:G' system

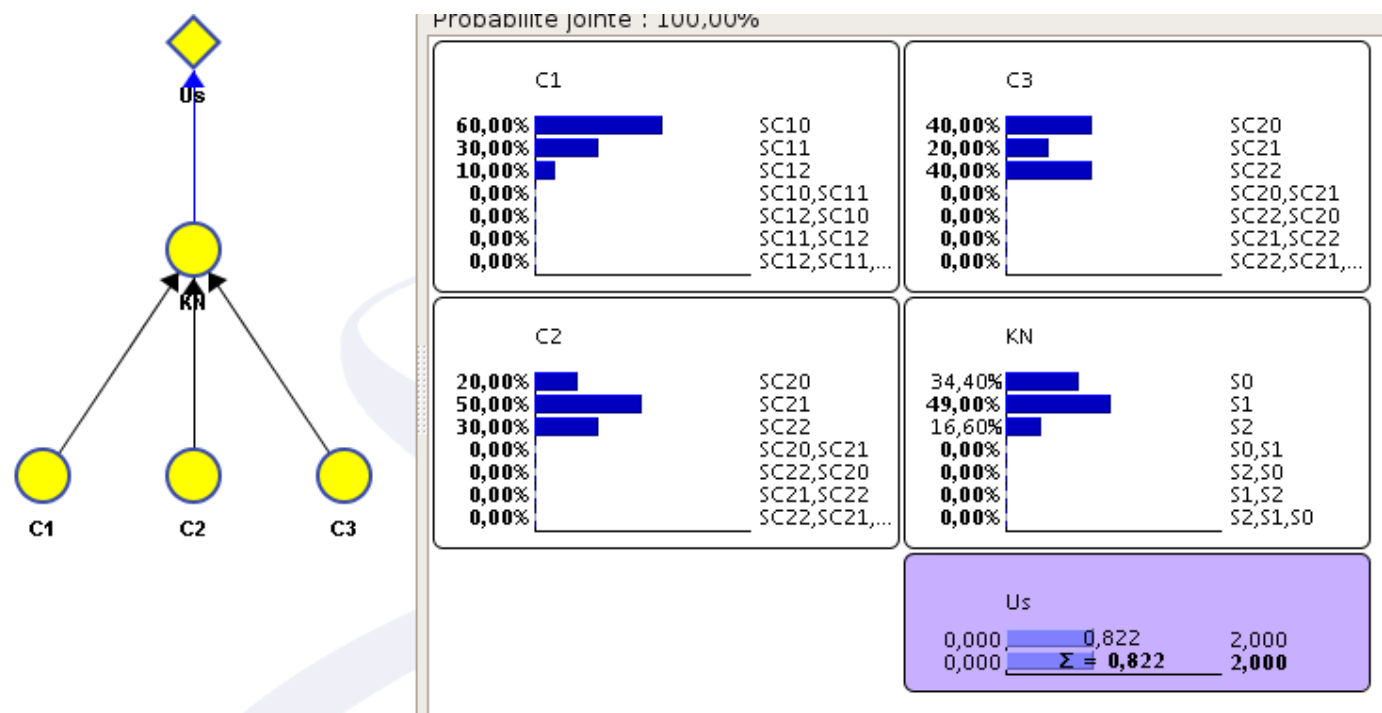

Fig. 8. Evidential network solution of [79]

with

$$
\begin{aligned}
\phi\left(x_{C_{i}}\right)=\phi & (([0.05,0.15],[0.25,0.35],[0.55,0.65]), \\
& ([0.25,0.35],[0.45,0.55],[0.15,0.25]), \\
& ([0.35,0.45],[0.15,0.25],[0.35,0.45])))
\end{aligned}
$$

The evidential network on figure 9 models the problem of imprecise probabilities and allows the computation of the system performance with its imprecision.

The computation nodes of $\mathrm{Bel}$ and $\mathrm{Pls}$ measures on system operating modes $s_{1}$ and $s_{2}$ were added to show that the bounding property of the values of probabilities in the precise case (previous section) is verified. The utility function integrated in the evidential network on figure 8 is now divided into two utility functions to compute upper limit $\bar{U}_{S}$ according to table XIII and lower limit $\underline{U}_{S}$ according to table XII as we proceed in section III-B.

As we can see on figure 9, the imprecision on the components probabilities to be in each operating modes is propagated through the network and induces a distribution of belief masses highlighting the imprecision on the system probability to be in its various operating modes. Taking into account the associated performances, the utility nodes introduced give a bound of
TABLE XII

UTILITY $\underline{U}_{S}$

\begin{tabular}{|c|c|}
\hline Focal set & utility \\
\hline \hline$s_{2}$ & 2 \\
$s_{1}$ & 1 \\
$s_{0}$ & 0 \\
$s_{2}, s_{1}$ & 1 \\
$s_{2}, s_{0}$ & 0 \\
$s_{1}, s_{0}$ & 0 \\
$s_{2}, s_{1}, s_{0}$ & 0 \\
\hline
\end{tabular}

TABLE XIII

UTILITY $\bar{U}_{S}$

\begin{tabular}{|c|c|}
\hline Focal set & utility \\
\hline \hline$s_{2}$ & 2 \\
$s_{1}$ & 1 \\
$s_{0}$ & 0 \\
$s_{2}, s_{1}$ & 2 \\
$s_{2}, s_{0}$ & 2 \\
$s_{1}, s_{0}$ & 1 \\
$s_{2}, s_{1}, s_{0}$ & 2 \\
\hline
\end{tabular}

the real utility value. Thus, the utility previously specifies $U_{s}=0.822$ on figure 8 when the a priori belief masses are 


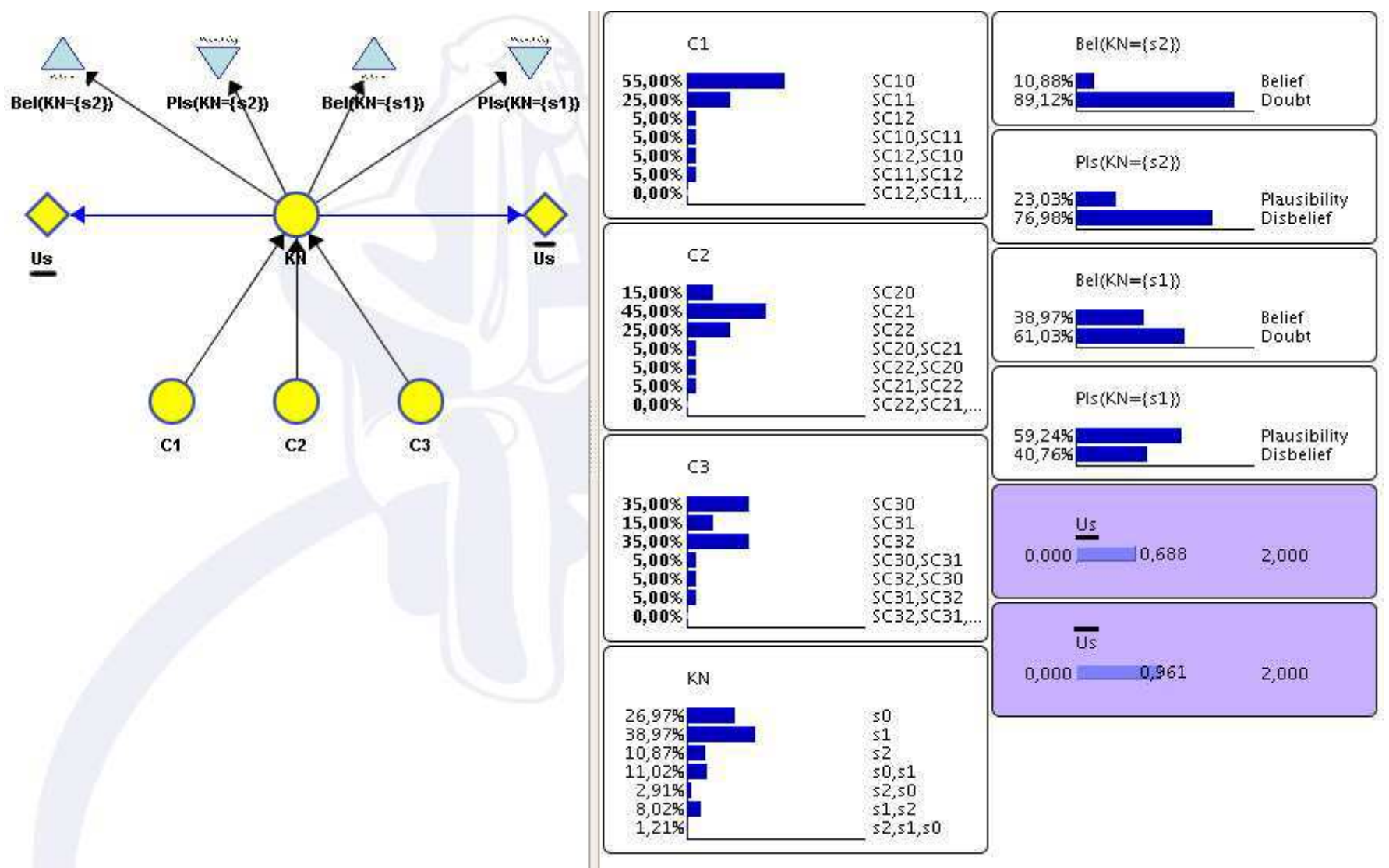

Fig. 9. EN to evaluate imprecise performance of a multistate system [79]

affected to the specific focal sets, is bounded by the lower and upper utilities $\left[\underline{U}_{S}, \bar{U}_{s}\right]=[0.688,0.961]$ as can be observed on figure 9. The bounding property is checked because we took care to bound each a priori belief mass of the problem without imprecision to deal with the problem with imprecision where we took a variation of \pm 0.05 . We can notice that the symmetrical bounding of the a priori belief masses does not necessarily give a symmetrical bounding of the utility.

3) Conclusion: The imprecision is propagated through the evidential network as well as in a problem of modeling of imprecise reliability of a system as for the performance evaluation by imprecise utility functions. This characteristic of evidential networks opens significant possibilities to model problems of reliability in a general way.

\section{Bride system: probist reliability}

For this second example, we have chosen a complex system in the reliability meaning as written by Villemeur [63] but with few components in order to facilitate the comprehension. The bridge system (cf. figure 10) was largely studied in the literature and Torres-Toledano [80] modeled its reliability with Bayesian networks. It consists of 5 components and each component has two disjoint states $(\{U p\},\{D o w n\})$ for a problem of probist reliability. The elementary events on these components are supposed to be independent. The system is homogeneous and no repair is considered.

By enumerating the minimal cuts or the minimal success paths, the evidential network shown on figure 11 is obtained

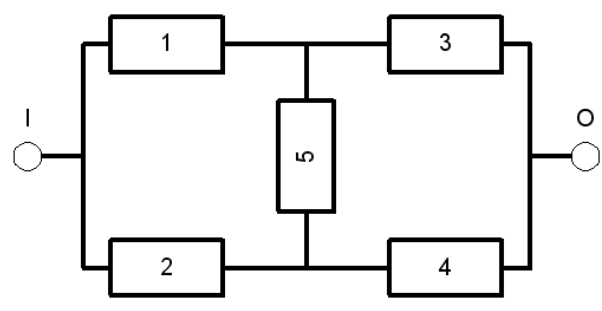

Fig. 10. Bridge system

to evaluate the reliability of the bridge system. This model was studied in [22].

1) Precise Probabilities: To study the reliability of this system, we first consider precise failure rates $\lambda_{1}=\lambda_{2}=\lambda_{5}=$ $10^{-3} h^{-1} ; \lambda_{3}=\lambda_{4}=2.10^{-3} h^{-1}$ and mission time $T_{i}=200 h$. The probabilities that each component is in state $\{U p\}$ are $P_{C_{I \mid I \in\{1,2,5\}}}(\{U p\})=0.81873$ and $P_{C_{J \mid J \in\{3,4\}}}(\{U p\})=0.67032$. By using 7, 8 and 13, the following a priori belief mass distributions are obtained:

$M_{C_{i}}=\left[\begin{array}{l}0.818730 .181270 \\ 0\end{array}\right]$ and $M_{C_{j}}=\left[\begin{array}{ll}0.67032 & 0.329680\end{array}\right]$

The propagation of a priori belief masses in the network gives system reliability $R=0.850134$ at $T_{i}=200 h$ as confirmed on figure 11. More details can be obtained in [22]

2) Imprecise Probabilities: Now, let us consider the same problem with imprecise failure rates expressed as intervals: $\left[\underline{\lambda}_{i}, \bar{\lambda}_{i}\right]=\left[0.9 e^{-3}, 1.1 e^{-3}\right]$ and $\left[\underline{\lambda}_{j}, \bar{\lambda}_{j}\right]=\left[1.9 e^{-3}, 2.1 e^{-3}\right]$. The a priori belief mass distribution defining the uncertain 


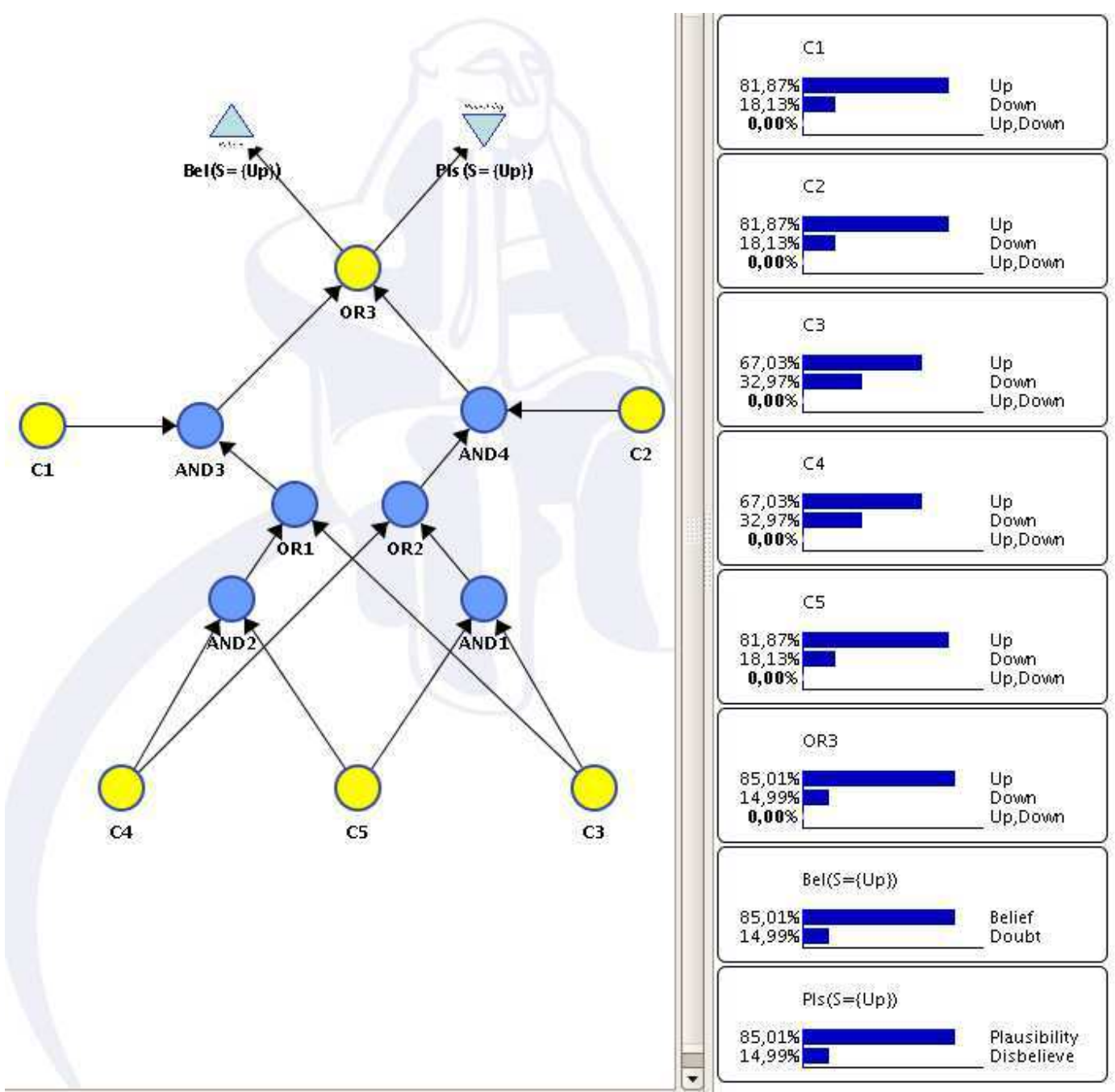

Fig. 11. Precise reliability of the bridge system

state of each component at mission time $T_{i}=200 h$ is obtained from 7,8 and 13 :

$$
\begin{aligned}
M_{C_{i \in\{1,2,5\}}} & =\left[\begin{array}{lll}
0.80252 & 0.16473 & 0.03275
\end{array}\right] \\
M_{C_{i \in\{3,4\}}} & =\left[\begin{array}{lll}
0.65705 & 0.31614 & 0.02681
\end{array}\right]
\end{aligned}
$$

Figure 12 shows the result obtained by the evidential network.

The system reliability is the probability that the system is in operating condition at time $T_{i}$. It is between $\left[\underline{R}\left(T_{i}\right), \bar{R}\left(T_{i}\right)\right]=$ $[0.83390 .8658]$. While comparing with the result of the previous section, we note that the bounding property (cf. 9) is verified. We can also note that the precise reliability obtained in section $\mathrm{V}-\mathrm{C} 1$ is not the center of the obtained p-box. It depends on the structure function modeled by the evidential network.

\section{Multistate bridge system}

In this section, we study the performance of the bridge system in the same way as proposed by [79] for the $k$-out-of$n: \mathrm{G}$ system studied in the previous section. We consider each component with 3 ordered levels of performance $\left\{u_{0}, u_{1}, u_{2}\right\}$ according to their operating mode. The structure function is a combination of 'AND' and 'OR' structure subfunctions of 2 components which are given by conditional belief mass table XIV and table XV).

TABLE XIV

'AND' STRUCTURE FUNCTION

\begin{tabular}{|c|c||c|}
\hline$C_{1}$ & $C_{2}$ & $\phi(x)$ \\
\hline \hline 0 & 0 & $s_{0}$ \\
0 & 1 & $s_{0}$ \\
0 & 2 & $s_{0}$ \\
1 & 0 & $s_{0}$ \\
1 & 1 & $s_{1}$ \\
1 & 2 & $s_{1}$ \\
2 & 0 & $s_{1}$ \\
2 & 1 & $s_{1}$ \\
2 & 2 & $s_{2}$ \\
\hline
\end{tabular}

The structure function of a 'AND' gate between two components with 3 states each (cf. table XIV) is converted into a conditional belief mass table (cf. table XVI). The same translation can be carried out for a 'OR' gate. These conditional belief mass tables can thus be used within the 


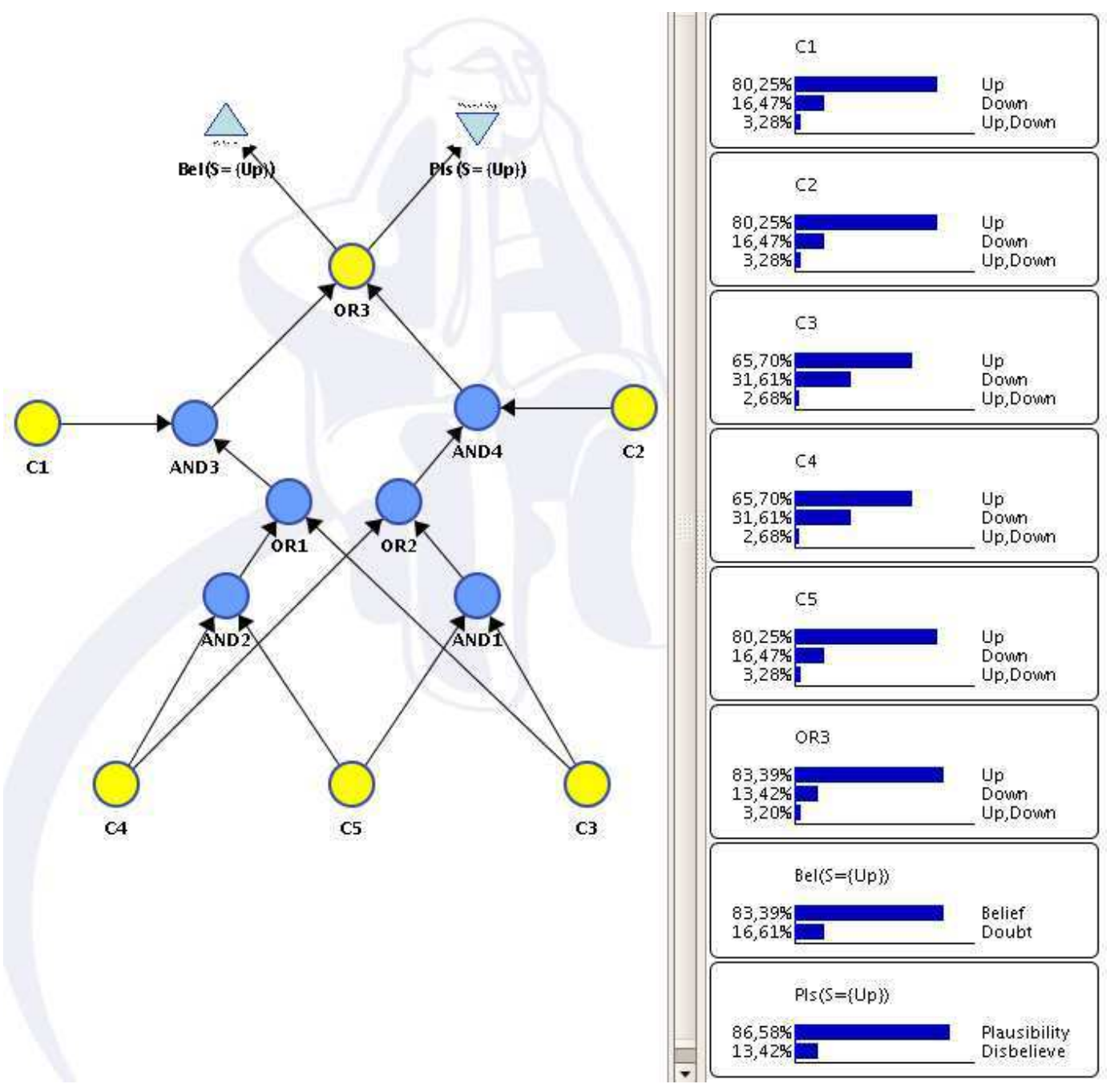

Fig. 12. Imprecise reliability of the bridge system

TABLE XV

'OR' STRUCTURE FUNCTION

\begin{tabular}{|c|c||c|}
\hline$C_{1}$ & $C_{2}$ & $\phi(x)$ \\
\hline \hline 0 & 0 & $s_{0}$ \\
0 & 1 & $s_{1}$ \\
0 & 2 & $s_{2}$ \\
1 & 0 & $s_{1}$ \\
1 & 1 & $s_{1}$ \\
1 & 2 & $s_{2}$ \\
2 & 0 & $s_{2}$ \\
2 & 1 & $s_{2}$ \\
2 & 2 & $s_{2}$ \\
\hline
\end{tabular}

evidential network if the combination of the operating modes of the components induces the operating mode of the system according to the suggested structure functions. It should be noted that other structure functions could be modeled.

1) Precise probabilities: Let us consider that the system components show probability set $P_{C_{i \mid i \in\{1,2,5\}}}=$ $\left[\begin{array}{lll}0.1 & 0.3 & 0.6\end{array}\right]$ and $P_{C_{i \mid i \in\{, 4\}}}=\left[\begin{array}{lll}0.3 & 0.5 & 0.2\end{array}\right]$ for performance levels $\left\{u_{2}, u_{1}, u_{0}\right\}$. As in section V-B1, we can compute the system utility from the belief mass distributions resulting from this set of probabilities and 7, 8 and
13. Thus, $M_{C_{I \mid I \in\{1,2,5\}}}=\left[\begin{array}{llllllll}0.1 & 0.3 & 0.6 & 0 & 0 & 0 & 0\end{array}\right]$ and $M_{C_{I \mid I \in\{3,4\}}}=$ $\left[\begin{array}{lllllll}0.3 & 0.5 & 0.2 & 0 & 0 & 0 & 0\end{array}\right]$ are obtained.

The utility tables for $\underline{U}_{S}$ and $\bar{U}_{S}$ are given by tables XII and XIII. The provided distributions are precise and the the system performance is also precise as shown on figure 13 with an utility $U_{S}=U_{S}=\bar{U}_{S}=0.631$.

2) Imprecise probabilities: Now, let us consider an imprecise set of probabilities concerning the distribution over the operating conditions of the components. Each probability is now imprecise with an inaccuracy of \pm 0.05 . The a priori belief mass distributions of the components are computed again by 7,8 and 13 :

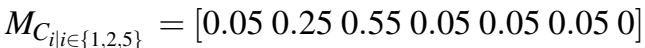

$$
\begin{aligned}
& M_{C_{i \mid i \in\{3,4\}}}=\left[\begin{array}{llll}
0.25 & 0.450 .150 .050 .05 & 0.050
\end{array}\right]
\end{aligned}
$$

All the a priori mass distributions, the measures of belief and plausibility of the system at level of performance $u_{2}$ and $u_{1}$ and utilities $\underline{U}_{S}, \bar{U}_{S}$ are indicated on figure 14 .

As in the previous example, the upper and lower bounds of the system utility surrounds the precise utility. Equation 9 is verified in the case of an utility computation. We notice that the system complexity is not an influence factor on the result 
obtained.

\section{CONCLUSION}

In this article, we address the problem of imprecision in the reliability and performance assessment of multi-state systems. For this purpose, we have proposed the use of the Dempster-Shafer theory to model the structure function of studied systems by a network approach.

As a first step, we remembered the basics of the DemspterShafer theory for the evaluation of reliability and performance. We have thus laid the groundwork for a model of evidential networks using junction trees and Bayesian inference extended to belief masses. We have also introduced the computation of utilities associated with these networks.

In a second step, we showed how to study the probist reliability of systems, regardless of their complexity, using evidential networks and in particular how to take into account the uncertainty about the failure rates. We showed that Bayesian evidences provide a precise reliability value even if they are coded in an evidential network, which allows a conventional predictive assessment of the reliability by fault trees or system performance by Markov chains.

When the input data is imprecise, we have a problem of imprecise probabilities and we identified that evidential networks were able to propagate this imprecision from the root nodes to the target nodes. This capability allows us to determine the uncertainty of the reliability or the system performance. In particular, we have shown that bounding precise input data by intervals of probabilities led to the bounding of the sought reliability or performance. This bounding allows us to compute optimistic and pessimistic values of the studied parameter.

Finally, we have shown how to model precise and imprecise reliability of a simple $k$-out-of- $n: \mathrm{G}$ system, then the precise and imprecise performance assessment of any system. Thus, we are able to show the equivalence with other models. Then, we have dealt with the reliability analysis of the bridge system, which is a small complex problem in the reliability sense. We have evaluated its precise and imprecise performance, which allowed us to conclude that evidential networks and associated utility functions allow an easy and powerful modeling for the study of predictive parameters of complex systems.

This modeling capability pointed out taht precise or imprecise reliability or performance assessments of systems can be extended to other parameters, and that evidential networks can address more general problems of imprecise knowledge.

\section{REFERENCES}

[1] ANSI/ISA-S84.01-1996, Application of Safety Instrumented Systems for the process control industry. Instrumentation Society of America (ISA), 1996.

[2] IEC61508, Functional safety of Electrical/Electronic/Programmable Electronic (E/E/PE) safety related systems. International Electrotechnical Commission (IEC), 1998.

[3] IEC61511, Functional safety: Safety Instrumented Systems for the process industry sector. International Electrotechnical Commission (IEC), 2000.

[4] IEC61025, IEC 61025: fault tree analysis. IEC, 1990.

[5] H. Wang and H. Pham, "Survey of reliability and availability evaluation of complex networks using monte carlo techniques," Microelectronic reliability, vol. 37, no. 2, pp. 187-209, 1997.
[6] K. D. Rao, H. Kushwaha, A. Verma, and A. Srividya, "Quantification of epistemic and aleatory uncertainties in level-1 probabilistic safety assessment studies," Reliability Engineering and System Safety, vol. 92, no. 7, pp. 947-956, 2007. [Online]. Available: http://www.sciencedirect.com/science/article/B6V4T-4KRY3MW2/2/29a81ca2a3dc6943447fe19706a1837b

[7] P. Weber and L. Jouffe, "Complex system reliability modelling with dynamic object oriented bayesian networks (doobn)." Reliability Engineering and System Safety, vol. 91, no. 2, pp. 149-162, February 2006.

[8] H. Langseth and L. Portinal, "Bayesian networks in reliability," Reliability Engineering and System Safety, vol. 92, no. 1, pp. 92-108, January 2007.

[9] A. Mittal, A. Kassim, and T. Tan, Bayesian Network Technologies: Applications and Graphical Models, A. Mittal and A. Kassim, Eds. IGI publishing, 2007.

[10] L. Utkin and F. Coolen, New metaheuristics, neural \& fuzzy techniques in reliability, ser. Computational intelligence in reliability engineering. G. Levitin, 2007, vol. 2, ch. Imprecise reliability: An introductory overview., pp. Chapter 10, pp. 261-306.

[11] I. Kozine and L. Utkin, "Interval valued finite markov chaines," Reliable computing, vol. 8, pp. 97-113, 2002.

[12] D. Coit, T. Jin, and N. Wattanapongsakorn, "System optimization with component reliability estimation uncertainty: A multicriteria approach," IEEE Transactions on Reliability, vol. 53, no. 3, pp. 369-380, September 2004.

[13] D. Berleant and J. Zhang, "Bounding the times to failure of 2-component systems," IEEE Transactions on Reliability, vol. 53, no. 4, pp. 542-550, 2004.

[14] F. Coolen and L. Utkin, "Imprecise reliability: A concise overview," in Risk, Reliability and Societal Safety, T. Aven and J. Vinnem, Eds. Stavanger: Taylor \& Francis Group, June 2007, pp. 1959-1966.

[15] K.-Y. Cai, "System failure engineering and fuzzy methodology: An introductory overview," Fuzzy Sets and Systems, vol. 83, no. 2, pp. 113-133, 1996. [Online]. Available: http://www.sciencedirect.com/science/article/B6V05-3VTK28C$1 / 2 / 43 \mathrm{cc} 02 \mathrm{c} 0 \mathrm{c} 47 \mathrm{c} 1099 \mathrm{a} 7 \mathrm{c} 4 \mathrm{~d} 9 \mathrm{a} 62 \mathrm{c} 238594$

[16] H. Tanaka, C. Fan, F. Lai, and K. Toguchi, "Fault tree analysis by fuzzy probability," IEEE Transactions on Reliability, vol. 32, pp. 453-457, 1983.

[17] D. Singer, "A fuzzy set approach to fault tree and reliability analysis," Fuzzy Sets and Systems, vol. 34, pp. 145-155, 1990.

[18] M. Sallak, C. Simon, and J.-F. Aubry, "A fuzzy probabilistic approach for determining safety integrity level," IEEE Transactions on Fuzzy Systems, 2007

[19] L. Guo, "Software quality and reliability prediction using dempstershafer theory," Ph.D in computer and information science, College of Engineering and Mineral Resources at West Virginia University, Lane Department of Computer Science and Electrical Engineering, Morgantown, West Virginia, 2004.

[20] S. Ferson, C. A. Joslyn, J. C. Helton, W. L. Oberkampf, and K. Sentz, "Summary from the epistemic uncertainty workshop: consensus amid diversity," Reliability Engineering \& System Safety, vol. 85, no. Issues 1-3, pp. 355-369, 2004.

[21] C. Simon, P. Weber, and E. Levrat, "Bayesian networks and evidence theory to model complex systems reliability," Journal of Computers, vol. 2, no. 1, pp. 33-43, February 2007. [Online]. Available: http://www.academypublisher.com/jcp/vol02/no01/jcp02013343.pdf

[22] C. Simon, P. Weber, and A. Evsukoff, "Bayesian networks inference algorithm to implement dempster shafer theory in reliability analysis," Reliability Engineering and System Safety, vol. 93, no. 7, pp. 950-963, July 2008.

[23] P. Shenoy, Valuation based systems: a framework for managing uncertainty in expert systems. Wiley, New-York, 1992, pp. 83-104.

[24] R. Demirer and P. P. Shenoy, "Sequential valuation networks: A new graphical technique for asymmetric decision problems," in ECSQARU 01: Proceedings of the 6th European Conference on Symbolic and Quantitative Approaches to Reasoning with Uncertainty. London, UK: Springer-Verlag, 2001, pp. 252-265.

[25] B. R. Cobb and P. P. Shenoy, "A comparison of bayesian and belief function reasoning," University of Kansas, School of Business, Tech. Rep. 292, September 2002.

[26] P. Smets, TBMLAB, IRIDIA, Brussels, Belgium, March 2004

[27] F. Cozman, "Credal networks," Artificial Intelligence Journal, vol. 120, pp. 199-233, 2000.

[28] — , "Graphical models for imprecise probabilities," International Journal of Approximate Reasoning, vol. 39, no. 2-3, pp. 167-184, 2005 
[29] B. B. Yaghlane and K. Mellouli, "Inference in directed evidential networks based on the transferable belief model," International Journal of Approximate Reasoning, 2008.

[30] A. P. Dempster, "Upper and lower probabilities induced by a multivalued mapping," Annals of Mathematical Statistics, vol. 38, pp. 325-339, 1967.

[31] G. Shafer, A Mathematical Theory of Evidence. Princeton University Press, 1976.

[32] P. Walley, Statistical reasoning with imprecise probabilities. Chapman and Hall, 1991.

[33] J. Kholas and P. Monney, a mathematical theory of hints: an approach to Dempster-Shafer theory of evidence. Springer Verlag, 1995, vol. 425.

[34] P. Smets, "Belief induced by the knowledge of some probabilities," in Uncertainty in AI'94, D. Heckerman, D. Poole, and R. L. de Mantaras, Eds. San Mateo, California: Morgan Kaufmann, 1994, pp. 523-530.

[35] K. Sentz and S. Ferson, "Combination of evidence in dempster-shafer theory," Sandia National Laboratory, Tech. Rep., 2002.

[36] E. Ouzounova, "Incertitude et mesure de performance: Une nouvelle approche travers la thorie mathmatique des indications," Ph.D. dissertation, Facult des sciences conomiques et sociales, Universit de Fribourg, Suisse, 2005.

[37] M. H. Duong, "Modle de prcaution en conomie: introduction aux probabilits imprcises," Ph.D. dissertation, Universit de Paris I- Panthon Sorbonne, 2005

[38] G. J. Klir and T. A. Folger, Fuzzy sets, uncertainty, and information. Upper Saddle River, NJ, USA: Prentice-Hall, Inc., 1987.

[39] B. A. Chokr and V. Y. Kreinovich, Advances in the Dempster-Shafer theory of evidence. New York, NY, USA: John Wiley \& Sons, Inc., 1994 , ch. How far are we from the complete knowledge? Complexity of knowledge acquisition in the Dempster-Shafer approach, pp. 555-576.

[40] A.-O. Boudraa, A. Bentabet, F. Salzenstei, and L. Guillon, "Dempstershafer's basic probability assignment based on fuzzy membership functions," Electronic Letters on Computer Vision and Image Analysis, vol. 4 no. 1, pp. 1-9, 2004

[41] I. Gilboa and D. Schmeidler, "Maximin expected utility with non-unique prior," Journal of Mathematical economics, vol. 18, pp. 141-153, 1989.

[42] G. Klir and M. Wierman, "Uncertainty-based information. elements of generalized information theory," in Studies in Fuzziness and SoftComputing. Physica-Verlag, 1998, p. 168.

[43] P. Smets and R. Kennes, "The transferable belief model," Artificial Intelligence, vol. 66, pp. 191-243, 1994.

[44] G. Klir and B. Yuan, Fuzzy Sets and Fuzzy Logic. Theory and Applications., U. S. River, Ed. NJ:Prentice Hall, 1995.

[45] M. Guth, "A probability foundation for vagueness and imprecision in fault tree analysis," IEEE Transactions on Reliability, vol. 40, no. 5, pp. 563-570, 1991.

[46] Y. Cheng, "Uncertainty in fault tree analysis," Tamkang Journal of Science and Engineering, vol. 3, no. 1, pp. 23-29, 2000.

[47] J. Hall and J. Lawry, "Imprecise probabilities of engineering system failure from random and fuzzy set reliability analysis," in ISIPTA'01, 26-29 june 2001.

[48] U. K. Rakowsky, "Fundamentals of the dempster-shafer theory and its applications to system safety and reliability modelling," in Proceedings of the ESRA Summer Safety and Reliability Seminars - SSARS 2007, Sopot, Poland, July 2007.

[49] P. Smets, "The application of the matrix calculus to belief functions," Int. J. Approximate Reasoning, vol. 31, pp. 1-30, 2002.

[50] S. Ferson, R. B. Nelsen, J. Hajagos, D. J. Berleant, J. Zhang, W. T. Tucker, L. R. Ginzburg, and W. L. Oberkampf, "Dependence in probabilistic modeling, dempster-shafer theory, and probability bounds analysis," Sandia National Laboratory, Tech. Rep. 3072, 2004.

[51] H. Regan, S. Ferson, and D. Berleant, "Equivalence of five methods for bounding uncertainty." International Journal of Approximate Reasoning, vol. 36, pp. 1-30, 2004

[52] C. Joslyn and S. Ferson., "Approximate representations of random intervals for hybrid uncertainty quantification in engineering modeling," in Proceedings of the Fourth International Conference on Sensitivity Analysis of Model Output., Los Alamos National Laboratory, Los Alamos, NM., 2004.

[53] S. Ferson, V. Kreinovich, L. Ginzburg, D. Myers, and K. Sentz, "Constructing probability boxes and dempster-shafer structures," Sandia National Laboratory, Tech. Rep., 2002.

[54] P. Smets, "The transferable belief model and other interpretations of dempster-shafer's model," in UAI '90: Proceedings of the Sixth Annual Conference on Uncertainty in Artificial Intelligence. New York, NY, USA: Elsevier Science Inc., 1991, pp. 375-384.
[55] F. Jensen, An Introduction to Bayesian Networks, U. Press, Ed. London: UCL Press, 1996.

[56] J. von Neumann and O. Morgenstern, Theory of games and economic behavior. Princeton University Press, Princeton, 1944, second edition in 1947 , third in 1954.

[57] S. Russell and P. Norvig, Artificial intelligence: a modern approach, 2nd Edition. Prentice Hall., 2003, 2nd Edition.

[58] T. Paek and E. Horvitz, "On the utility of decision theoretic hidden subdialog." in Proceedings of International Speech Communication Association (ISCA), ser. Workshop on Error Handling in Spoken Dialogue Systems., Chateaux d'Oex, Switzerland, 2003, pp. 95-100.

[59] J.-Y. Jaffray, "Linear utility theory for belief functions," Operations Research Letters, vol. 8, pp. 107-112, 1989.

[60] G. Cooper, "The computational complexity of probabilistic inference using bayesian belief networks (research note)," Artif. Intell., vol. 42 no. 2-3, pp. 393-405, 1990.

[61] A. Bobbio, L. Portinale, M. Minichino, and E. Ciancamerla, "Improving the analysis of dependable systems by mapping fault trees into bayesian networks," Reliability Engineering and System Safety, vol. 71, no. 3, pp. 249-260, March 2001.

[62] H. Boudali and J. Dugan, "A discrete-time bayesian network reliability modeling and analysis framework," Reliability Engineering and System Safety, vol. 87, pp. 337-349, 2005.

[63] A. Villemeur, Reliability, availability, maintainability and safety assessment: methods and techniques. Wiley, 1992.

[64] OREDA, Offshore reliability data handbook, 4th Edition. Sintef, 2002.

[65] Exida, Safety Equipment Reliability Handbook, 2nd Edition. Exida, 2005.

[66] S. Hauge, H. Langseth, and T. Onshus, Reliability Data for Safety Instrumented Systems, PDS Data Handbook. Sintef, 2006.

[67] W. M. Goble and H. Cheddie, Safety Instrumented Systems VerificationPractical Probabilistic Calculations. ISA, 2006.

[68] T. A. Kletz, HAZOP and HAZAN: Identifying and assessing process industry hazards, 4th edition. Institution of chemical engineers, 1999.

[69] MIL217B, Reliability Prediction of Electronic Equipment. Dept. of Defence, USA, 1974.

[70] IEEE, IEEE guide to the collection and presentation of electrical, electronic, sensing component, and mechanical equipement reliability data for nuclear-power generating station. IEEE-std-500, 1984.

[71] CCPS, Guidelines for process equipement reliability data with data tables. Center for Chemical Process Safety/AIChE, 1991.

[72] _ Offshore reliability data handbook, 4th Edition. SINTEF, 2002.

[73] G. Levitin, "Block diagram method for analyzing multi-state systems with uncovered failures," Reliability Engineering and System Safety, vol. 92, pp. 727-734, 2007.

[74] K.-Y. Cai, C.-Y. Wen, and M.-L. Zhang, "Mixture models in profust reliability theory," Microelectronics and Reliability, vol. 35, no. 6, pp. 985-993, June 1995. [Online]. Available: http://www.sciencedirect.com/science/article/B6V47-3YMWKJN28/2/8c1da3873ad4e96308493a21ee01f895

[75] X. T. Hong-Zhong Huang and M. J. Zuo, "Posbist fault tree analysis of coherent systems," Reliability Engineering and System Safety, vol. 84, no. 2, pp. 141-148, May 2004. [Online]. Available: http://www.sciencedirect.com/science/article/B6V4T4BCXJJ6-1/2/4fcc742ab3f95a9281f3951ce5d52f3e

[76] H.-C. Wu, "Fuzzy reliability estimation using bayesian approach," Computers and Industrial Engineering, vol. 46, pp. 467-493, 2004.

[77] D. Pandey and S. Tyagi, "Profust reliability of a gracefully degradable system," Fuzzy Sets and Systems, vol. 158, pp. 794-803, 2007.

[78] O. Pourret, J. Collet, and J.-L. Bonouzounaova, "Evaluation of the unavailability of a multistate-component system using a binary model," Reliability Engineering and System Safety, vol. 64, pp. 13-17, 1999.

[79] G. Gopal, "Schur property of the performance function for the multistate coherent system," in Proceedings of the Third International conference on Mathematical Methods in Reliability, H. Langseth and B. Lindqvist, Eds., 2002, pp. 255-258. [Online]. Available: citeseer.ist.psu.edu/526472.html

[80] J. Torres-Toledano and L. Sucar, "bayesian networks for reliability of complex systems," in Progress in Artificial Intelligence, IBERAMIA'98, H. Coelho, Ed. Springer-Verlag, 2004, pp. 195-206. 


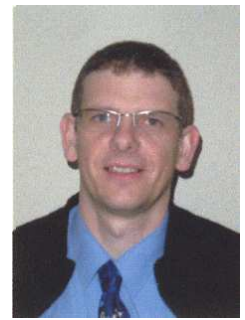

C hristophe SIMON received both the M.S. degree in Metrology, Control Systems and Electrotechnic in 1991 and the Ph.D. degree in 1996 from the University Henri Poincaré - Nancy 1, France. In 1999, he joined the Department of Quality, Industrial Logistic and Organization, IUT Epinal, at the University of Nancy 2, France, as an Assistant Professor. In 1992, he joined the Research Center for Automatic Control, Nancy, France. His research area concerns reliability and systems safety, pattern recognition, non-additive theories. Dr. Simon is a member of the French Association of Electrical, Electronic and System Control (Club EEA).

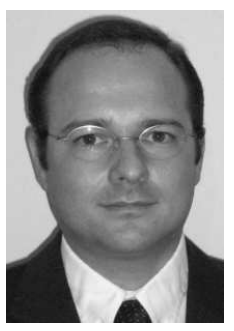

P hilippe Weber received his $\mathrm{PhD}$ degree in electrical engineering from the University of Grenoble, his MS degree in electrical engineering from the University of Nancy 1 in 1993. He is currently an Assistant Professor of RAMS, in the ESSTIN. 
TABLE VII

CONDITIONAL BELIEF MASS TABLE OF A '2-OUT-OF-3:G' GATE

\begin{tabular}{|c|c|c|c|c|c|}
\hline$E_{V}$ & $E_{X}$ & $E_{Y}$ & $\{\mathrm{Up}\}$ & $\begin{array}{r}E_{Z} \\
\text { \{Down }\end{array}$ & $\{$ Up,Down $\}$ \\
\hline$\{\mathrm{Up}\}$ & $\{\mathrm{Up}\}$ & $\overline{\mathrm{U} U \mathrm{p}\}}$ & 1 & $\overline{0}$ & 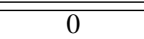 \\
\hline$\{$ Down $\}$ & $\{\mathrm{Up}\}$ & $\{\mathrm{Up}\}$ & 1 & 0 & 0 \\
\hline$\{$ Up,Down $\}$ & $\{\mathrm{Up}\}$ & $\{\mathrm{Up}\}$ & 1 & 0 & 0 \\
\hline$\{\mathrm{Up}\}$ & $\{$ Down & $\{\mathrm{Up}\}$ & 1 & 0 & 0 \\
\hline$\{$ Down & $\{$ Down $\}$ & $\{\mathrm{Up}\}$ & 0 & 1 & 0 \\
\hline$\{$ Up,Down $\}$ & $\{$ Down & $\{\mathrm{Up}\}$ & 0 & 0 & 1 \\
\hline$\{\mathrm{Up}\}$ & $\{$ Up,Down $\}$ & $\{\mathrm{Up}\}$ & 1 & 0 & 0 \\
\hline$\{$ Down & $\{$ Up,Down $\}$ & $\{\mathrm{Up}\}$ & 0 & 0 & 1 \\
\hline$\{$ Up,Down $\}$ & $\{$ Up, Down $\}$ & $\{\mathrm{Up}\}$ & 0 & 0 & 1 \\
\hline$\{\mathrm{Up}\}$ & $\{\mathrm{Up}\}$ & $\{$ Down $\}$ & 1 & 0 & 0 \\
\hline$\{$ Down $\}$ & $\{\mathrm{Up}\}$ & $\{$ Down $\}$ & 0 & 1 & 0 \\
\hline$\{$ Up,Down $\}$ & $\{\mathrm{Up}\}$ & $\{$ Down & 0 & 0 & 1 \\
\hline$\{\mathrm{Up}\}$ & $\{$ Down $\}$ & $\{$ Down $\}$ & 0 & 1 & 0 \\
\hline$\{$ Down & $\{$ Down & $\{$ Down & 0 & 1 & 0 \\
\hline$\{$ Up,Down $\}$ & $\{$ Down $\}$ & $\{$ Down $\}$ & 0 & 1 & 0 \\
\hline$\{\mathrm{Up}\}$ & $\{$ Up,Down $\}$ & $\{$ Down $\}$ & 1 & 0 & 0 \\
\hline$\{$ Down $\}$ & $\{$ Up,Down $\}$ & $\{$ Down $\}$ & 0 & 0 & 1 \\
\hline$\{$ Up,Down $\}$ & $\{\mathrm{Up}$, Down $\}$ & $\{$ Down $\}$ & 0 & 0 & 1 \\
\hline$\{\mathrm{Up}\}$ & $\{\mathrm{Up}\}$ & $\{$ Up,Down $\}$ & 1 & 0 & 0 \\
\hline$\{$ Down $\}$ & $\{\mathrm{Up}\}$ & $\{$ Up,Down $\}$ & 0 & 0 & 1 \\
\hline$\{$ Up,Down $\}$ & $\{\mathrm{Up}\}$ & $\{$ Up,Down $\}$ & 0 & 0 & 1 \\
\hline$\{\mathrm{Up}\}$ & $\{$ Down & $\{$ Up,Down $\}$ & 0 & 0 & 1 \\
\hline$\{$ Down $\}$ & $\{$ Down $\}$ & $\{$ Up,Down $\}$ & 0 & 1 & 0 \\
\hline$\{$ Up,Down $\}$ & $\{$ Down $\}$ & $\{$ Up,Down $\}$ & 0 & 0 & 1 \\
\hline$\{\mathrm{Up}\}$ & $\{$ Up,Down $\}$ & $\{$ Up,Down $\}$ & 1 & 0 & 0 \\
\hline$\{$ Down $\}$ & $\{$ Up,Down $\}$ & $\{$ Up,Down $\}$ & 0 & 0 & 1 \\
\hline$\{$ Up,Down $\}$ & $\{$ Up,Down $\}$ & $\{$ Up,Down $\}$ & 0 & 0 & 1 \\
\hline
\end{tabular}
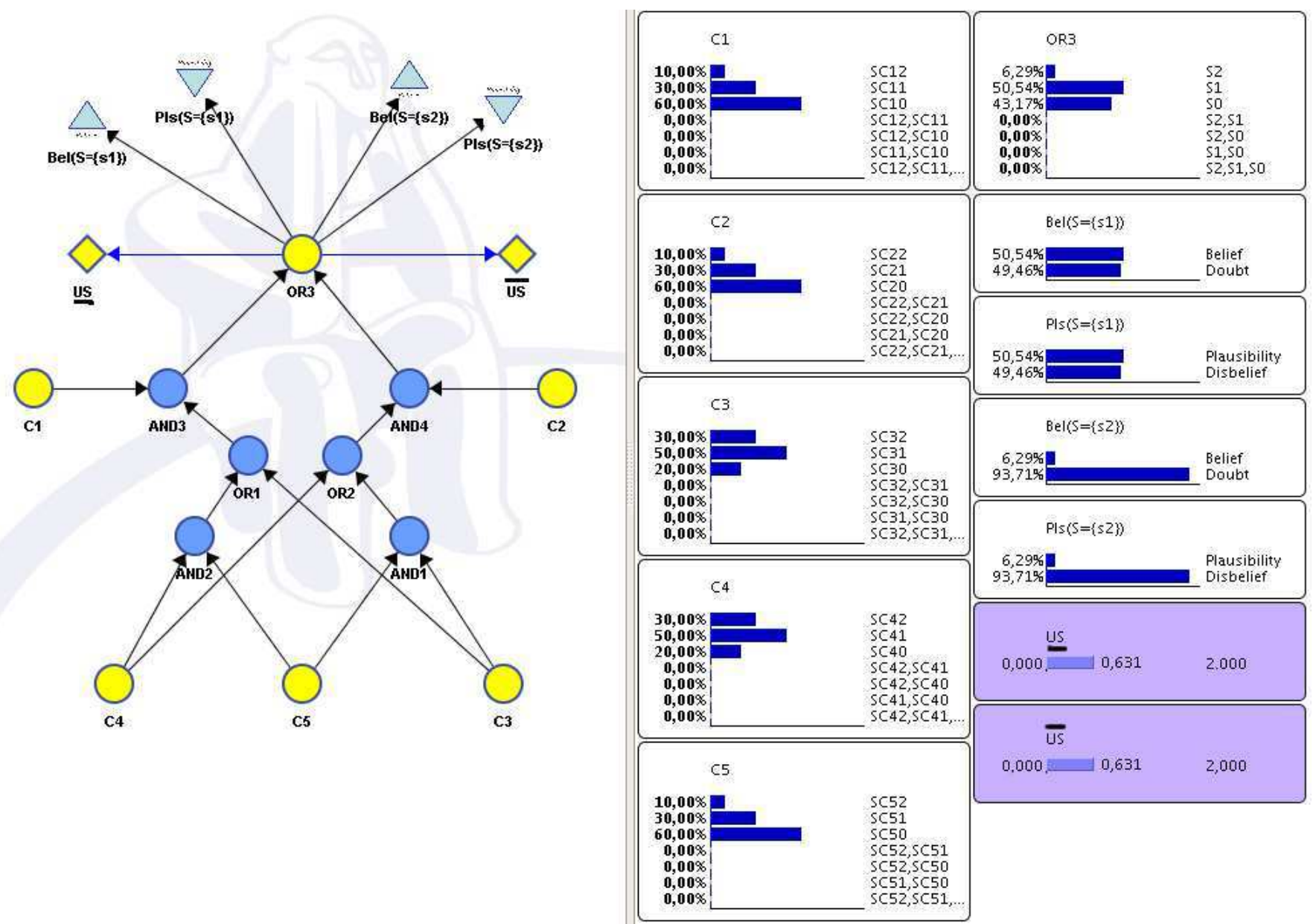

Fig. 13. Evidential network to evaluate the bridge system performance 
TABLE XVI

CONDITIONAL BELIEF MASS TABLE OF A 'AND' GATE WITH 3 STATES

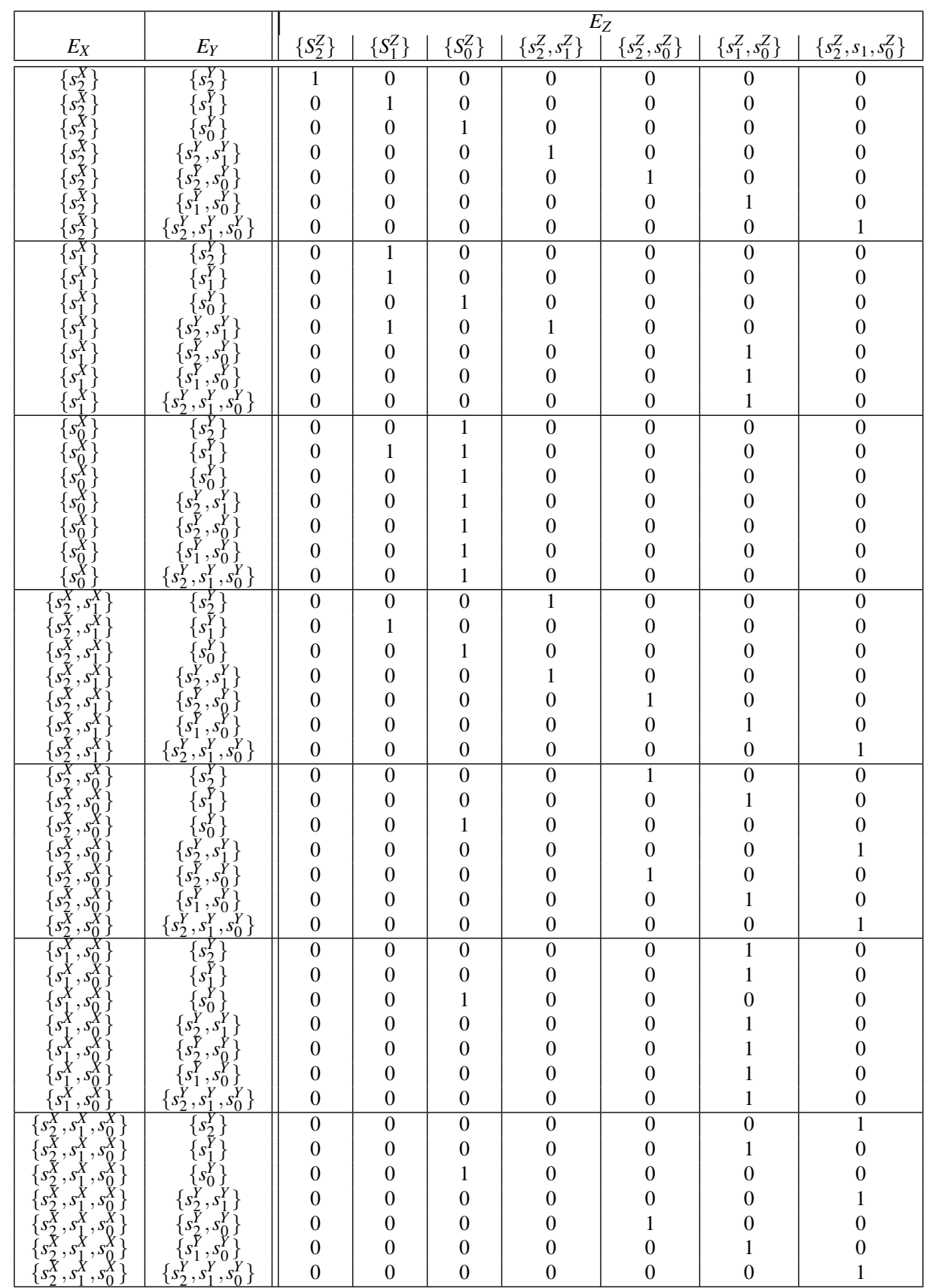




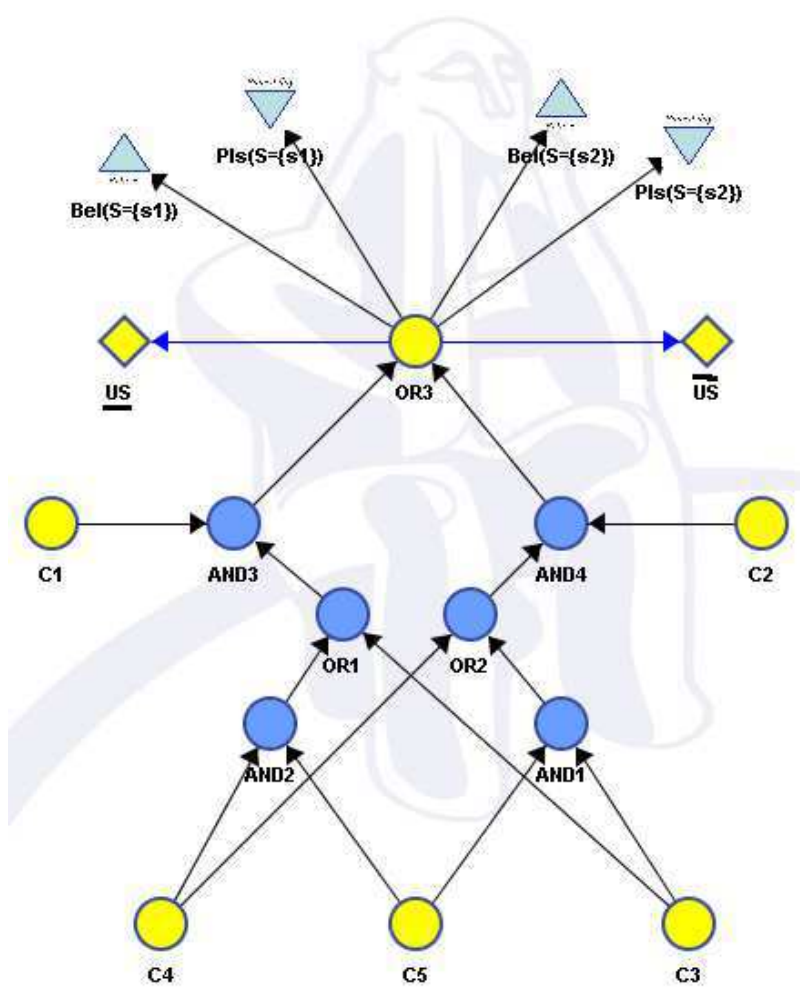

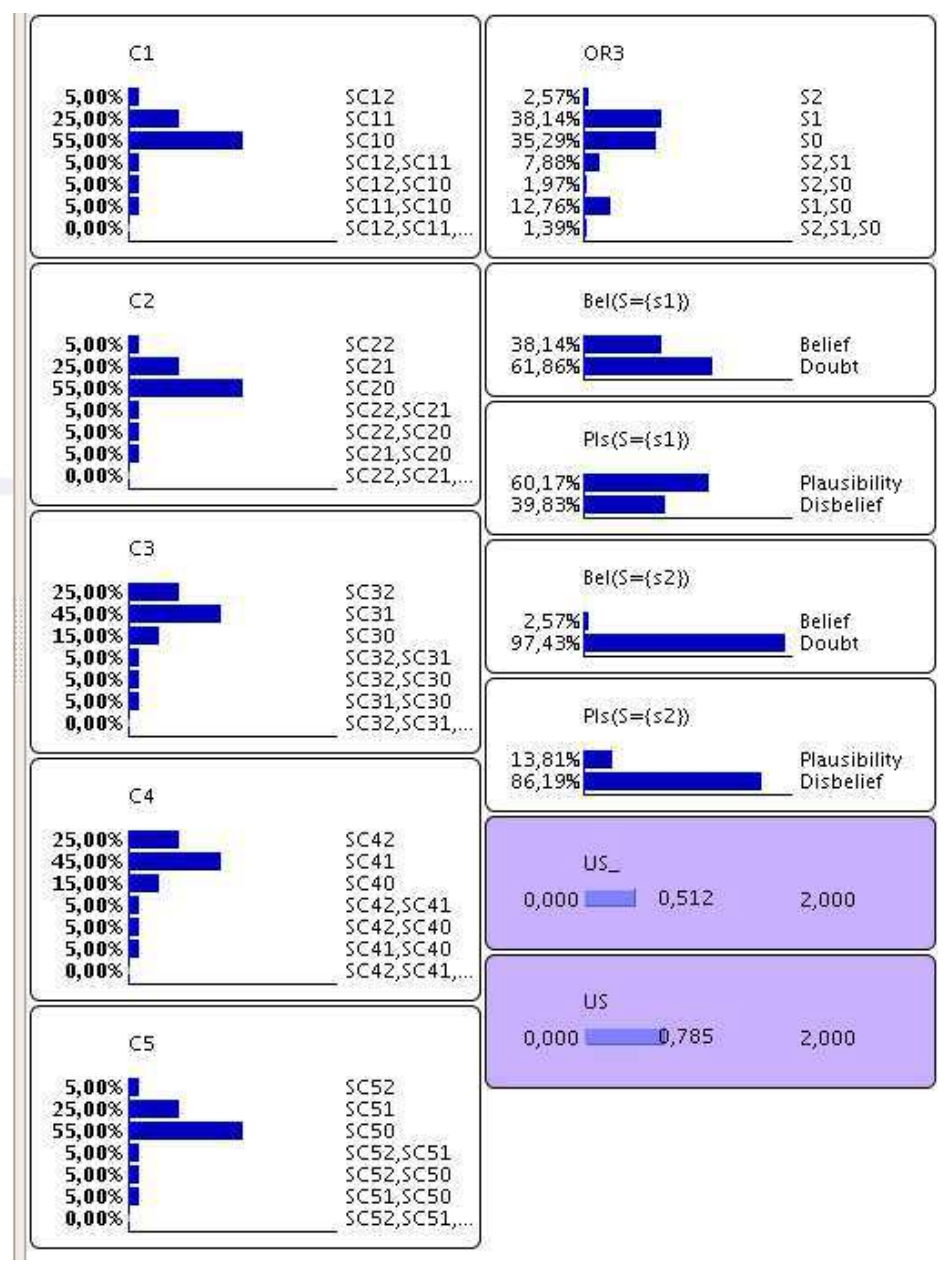

Fig. 14. Imprecise performance evaluation of the bridge system 